\title{
Altering Effort Costs in Parkinson's Disease with Noninvasive Cortical Stimulation
}

\author{
Yousef Salimpour, ${ }^{1}$ Zoltan K. Mari, ${ }^{2}$ and Reza Shadmehr ${ }^{1}$ \\ ${ }^{1}$ Laboratory for Computational Motor Control, Department of Biomedical Engineering, and ${ }^{2}$ Department of Neurology, Johns Hopkins School of Medicine, \\ Baltimore, Maryland 21205
}

In Parkinson's disease (PD), the human brain is capable of producing motor commands, but appears to require greater than normal subjective effort, particularly for the more-affected side. What is the nature of this subjective effort and can it be altered? We used an isometric task in which patients produced a goal force by engaging both arms, but were free to assign any fraction of that force to each arm. The patients preferred their less-affected arm, but only in some directions. This preference was correlated with lateralization of signaldependent noise: the direction of force for which the brain was less willing to assign effort to an arm was generally the direction for which that arm exhibited greater noise. Therefore, the direction-dependent noise in each arm acted as an implicit cost that discouraged use of that arm. To check for a causal relationship between noise and motor cost, we used bilateral transcranial direct current stimulation of the motor cortex, placing the cathode on the more-affected side and the anode on the less-affected side. This stimulation not only reduced the noise on the more-affected arm, it also increased the willingness of the patients to assign force to that arm. In a $3 \mathrm{~d}$ double-blind study and in a $10 \mathrm{~d}$ repeated stimulation study, bilateral stimulation of the two motor cortices with cathode on the more-affected side reduced noise and increased the willingness of the patients to exert effort. This stimulation also improved the clinical motor symptoms of the disease.

Key words: motor costs; Parkinson's disease; signal-dependent noise; tDCS

\section{Significance Statement}

In Parkinson's disease, patients are less willing to assign force to their affected arm. Here, we find that this pattern is direction dependent: directions for which the arm is noisier coincide with directions for which the brain is less willing to assign force. We hypothesized that if we could reduce the noise on the affected arm, then we may increase the willingness for the brain to assign force to that arm. We found a way to do this via noninvasive cortical stimulation. In addition to reducing effort costs associated with the affected arm, the cortical stimulation also improved clinical motor symptoms of the disease.

\section{Introduction}

The very language that describes motor symptoms of Parkinson's disease (PD) highlights a subjective scaling of time and space: bradykinesia (slowness of movement), tachyphemia (cluttering of speech), and micrographia (smallness of handwriting). Under certain circumstances, however, some of these symptoms spontaneously improve. For example, when asked to reach as fast as possible to pick up a ball, patients reach slower than normal if the

\footnotetext{
Received May 12, 2015; revised July 27, 2015; accepted July 30, 2015.

Author contributions: Y.S. and R.S. designed research; Y.S. performed research; Z.K.M. contributed unpublished reagents/analytic tools; Y.S. and R.S. analyzed data; Z.K.M. and R.S. wrote the paper.

This work was supported by the National Institutes of Health (Grant NS078311) and the Human Frontiers Science Program.

The authors declare no competing financial interests.

Correspondence should be addressed to Reza Shadmehr, Laboratory for Computational Motor Control, Department of Biomedical Engineering, 410 Traylor Building, Johns Hopkins School of Medicine, 720 Rutland Avenue, Baltimore, MD 21205. E-mail: shadmehr@jhu.edu.

DOI:10.1523/JNEUROSCI.1827-15.2015

Copyright $\odot 2015$ the authors $\quad 0270-6474 / 15 / 3512287-16 \$ 15.00 / 0$
}

ball is stationary, but at near normal speed if the ball is moving (Majsak et al., 1998). In particular, when the movement has increased urgency, for example, to prevent the ball from falling to the floor, the reach becomes even faster (Ballanger et al., 2006). Therefore, it is not that the PD brain is incapable of producing fast movements, it is as if the PD brain requires a greater than normal implicit motivation to produce the faster movements (Mazzoni et al., 2007). This has led to the hypothesis that, because faster and larger movements require greater force, perhaps the cost of generating force is higher than normal in PD. That is, the slowness and smallness of movements are not due to an abnormal scaling of time and space, but rather an elevated subjective cost of effort.

This idea is consistent with motor behaviors of animals that are challenged by altered levels of dopamine. For example, dopamine antagonists make rats less willing to exert effort (press levers) to acquire a valuable food (Salamone et al., 1991; Correa et al., 2002), whereas dopamine-enhancing agents make rats (Floresco et al., 2008; Bardgett et al., 2009) and humans (Wardle et al., 2011) more willing to exert effort. 
Table 1. Patient characteristics

\begin{tabular}{|c|c|c|c|c|c|c|c|c|c|c|c|c|c|}
\hline SID & Age & Sex & $\begin{array}{l}\text { Dominant } \\
\text { side }\end{array}$ & $\begin{array}{l}\text { Affected } \\
\text { side }\end{array}$ & Duration (y) & $\begin{array}{l}\text { UPDRS } \\
\text { motor }\end{array}$ & $\mathrm{H} \& \mathrm{Y}$ & $\begin{array}{l}\text { Levodopa } \\
\text { (mg/d) }\end{array}$ & Experiment 1 & Experiment 2 & Experiment 3 & Experiment 4 & Experiment 5 \\
\hline P01 & 54 & $\mathrm{~F}$ & Right & Right & 6 & 16 & 1.5 & 500 & $\sqrt{ }$ & $\sqrt{ }$ & & & \\
\hline P02 & 66 & $\mathrm{~F}$ & Right & Right & 2 & 6 & 1 & 300 & $\sqrt{ }$ & J & & & \\
\hline P03 & 60 & $\mathrm{~F}$ & Right & Right & 14 & 13 & 2.5 & 400 & $\sqrt{ }$ & $\sqrt{ }$ & & & \\
\hline P04 & 50 & $M$ & Right & Right & 10 & 17 & 2 & 700 & $\sqrt{ }$ & J & $\sqrt{ }$ & $\sqrt{ }$ & J \\
\hline P05 & 52 & $M$ & Right & Right & 4 & 14 & 1.5 & 300 & $\sqrt{ }$ & $\sqrt{ }$ & & & \\
\hline P06 & 55 & $M$ & Right & Right & 15 & 21 & 2 & 1200 & $\sqrt{ }$ & $\sqrt{ }$ & $\sqrt{ }$ & $\sqrt{ }$ & J \\
\hline P07 & 69 & $\mathrm{~F}$ & Right & Right & 5 & 18 & 1 & 600 & $\sqrt{ }$ & $\sqrt{ }$ & & & \\
\hline P08 & 66 & $M$ & Right & Right & 2 & 11 & 2 & 450 & $\sqrt{ }$ & $\sqrt{ }$ & & J & \\
\hline P09 & 65 & $M$ & Right & Right & 5 & 19 & 2.5 & 300 & $\sqrt{ }$ & $\sqrt{ }$ & & & \\
\hline P10 & 59 & $M$ & Right & Right & 6 & 22 & 1.5 & 400 & $\sqrt{ }$ & $\sqrt{ }$ & & & \\
\hline P11 & 61 & $\mathrm{~F}$ & Right & Right & 12 & 24 & 2 & 400 & $\sqrt{ }$ & & & & \\
\hline P12 & 62 & $\mathrm{~F}$ & Right & Right & 5 & 16 & 1 & 600 & $\sqrt{ }$ & & & & \\
\hline P13 & 57 & $M$ & Right & Right & 5 & 12 & 2 & 300 & $\sqrt{ }$ & & $\sqrt{ }$ & & \\
\hline P14 & 49 & $M$ & Right & Right & 7 & 15 & 1.5 & 1000 & $\sqrt{ }$ & & $\sqrt{ }$ & & $\sqrt{ }$ \\
\hline P15 & 75 & $M$ & Right & Right & 20 & 31 & 3 & 450 & $\sqrt{ }$ & & $\sqrt{ }$ & & \\
\hline P16 & 58 & $\mathrm{~F}$ & Right & Right & 8 & 17 & 2 & 500 & & & $\sqrt{ }$ & & \\
\hline P17 & 60 & $M$ & Right & Right & 2 & 20 & 1 & 300 & & & $\sqrt{ }$ & & J \\
\hline P18 & 78 & $M$ & Right & Right & 3 & 12 & 1 & 300 & & & $\sqrt{ }$ & & \\
\hline P19 & 76 & $M$ & Right & Right & 12 & 26 & 2 & 1500 & & & $\sqrt{ }$ & $\sqrt{ }$ & $\sqrt{ }$ \\
\hline P20 & 59 & $\mathrm{~F}$ & Right & Left & 4 & 15 & 1.5 & 400 & & & & & J \\
\hline P21 & 68 & $\mathrm{~F}$ & Right & Left & 2 & 12 & 1 & 300 & & & & & $\sqrt{ }$ \\
\hline P22 & 58 & $\mathrm{~F}$ & Right & Right & 3 & 15 & 1 & 300 & & & $\sqrt{ }$ & & $\sqrt{ }$ \\
\hline P23 & 69 & $\mathrm{~F}$ & Right & Right & 12 & 23 & 2 & 300 & & & & $\sqrt{ }$ & \\
\hline P24 & 51 & $\mathrm{~F}$ & Right & Right & 6 & 47 & 2 & 400 & & & & $\sqrt{ }$ & \\
\hline P28 & 56 & $M$ & Right & Bilateral & 8 & 23 & 1 & 1500 & & & & $\sqrt{ }$ & \\
\hline P29 & 60 & $\mathrm{~F}$ & Right & Left & 6 & 11 & 1 & 800 & & & & $\sqrt{ }$ & \\
\hline P30 & 70 & M & Right & Right & 3 & 30 & 2 & 100 & & & & $\sqrt{ }$ & \\
\hline P31 & 52 & $\mathrm{~F}$ & Right & Right & 9 & 37 & 2.5 & 450 & & & & $\sqrt{ }$ & \\
\hline
\end{tabular}

SID, Subject identification number; $H \& Y$, Hoehn and Yahr scale.

Here, we designed an experiment to measure the subjective cost of force production in PD. Our design relied on the fact that PD is a lateralized disease that affects one side of the body more than the other, producing unilateral tremor, rigidity, and bradykinesia, among other symptoms. The symptoms are related to loss of dopamine in the nigrostriatal system predominantly on the striatum contralateral to the affected body side (Tatsch et al., 1997). Therefore, we hypothesized that the cost of force production may be elevated for the more-affected arm with respect to the less-affected arm.

To test our idea, we instructed our volunteers to produce force through bilateral activation of the arms, but left it up to them to decide how much force should be assigned to each arm (O'Sullivan et al., 2009; Salimpour and Shadmehr, 2014). We found that, although the patients preferred to produce force with the less-affected side, the pattern was direction dependent. Surprisingly, for some directions, the subjective cost for the affected side was entirely normal, whereas for other directions, it was abnormally large. This direction-dependent pattern of subjective cost was stable from day to day and appeared to be related to the direction-dependent pattern of signal-dependent noise in each arm (the slope of the relationship between SD of force as a function of mean force).

The origin of signal-dependent noise is poorly understood, but may be partly due to the anisotropic distribution of preferred direction of cells in the contralateral primary motor cortex: directions of force for which more cells are available in the motor cortex (Scott et al., 2001) tend to exhibit less noise in generation of force (Salimpour and Shadmehr, 2014). To test whether unimanual signal-dependent noise affected subjective cost of force production in $\mathrm{PD}$, we attempted to alter this noise. Using simul- taneous transcranial direct current stimulation (tDCS) of both motor cortices, we were able to alter the signal-dependent noise. Interestingly, this not only produced a reduction in the subjective cost of force production, it also improved the clinical symptoms of the disease.

\section{Materials and Methods}

Experiments. We performed five experiments and in each experiment recruited $\sim 10-15$ volunteers diagnosed with PD (Table 1 ). In addition, we recruited $n=15$ healthy right-handed volunteers (age $54 \pm 8.6$ years, mean \pm SD, 5 females). All participants were naive to the purpose of the experiment and gave written informed consent. In all experiments, the patients were on their normal schedule of medication. The characteristics of the patients are summarized in Table 1. We assessed handedness using the Edinburgh Inventory (Oldfield, 1971). The experimental protocol was approved by the Institutional Review Board of the Johns Hopkins School of Medicine.

Bimanual task. Each volunteer held the handles of two robotic arms, one in each hand (Fig. 1A). Each handle housed a force transducer. The task was to produce forces with each arm in such a way that the sum of the two force vectors matched a target force. The subjects were shown a $0.5 \mathrm{~cm}$ radius cursor, the position of which, represented by vector $\mathbf{x}$, depended on the forces that they produced by their left and right arms:

$$
\mathbf{x}=a\left(\mathbf{f}_{L}+\mathbf{f}_{R}\right)+\mathbf{x}_{o}
$$

In Equation 1, $\mathbf{x}_{o}$ is the center of the workspace. The scaling factor $a$ was set to $0.5 \mathrm{~cm} / \mathrm{N}$, implying that, if the two arms together produced $20 \mathrm{~N}$ of force, the cursor was displaced by $10 \mathrm{~cm}$. The robots countered the forces at the handle by a stiff force field (stiffness of $2000 \mathrm{~N} / \mathrm{m}$, viscosity of 40 $\mathrm{N} / \mathrm{s} / \mathrm{m}$ ) that maintained each handle in a virtual well, minimizing but not eliminating movements. The robot sensors measured hand position and force at $200 \mathrm{~Hz}$. 

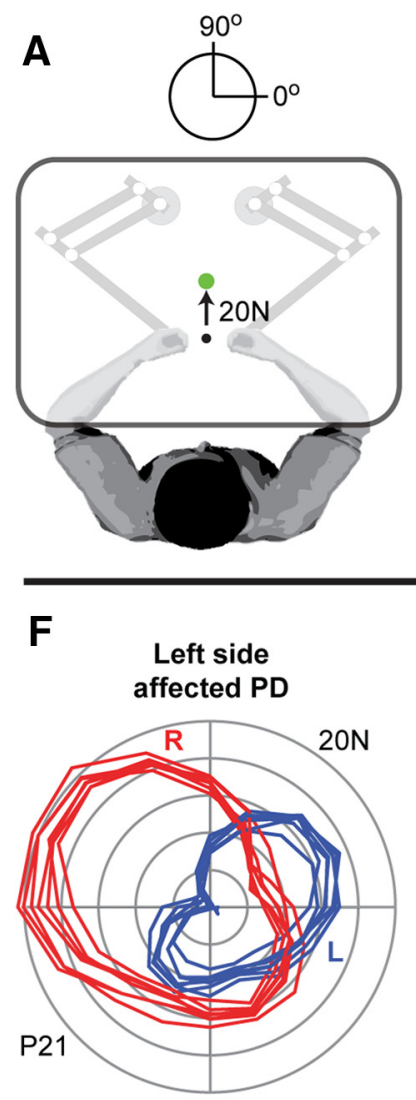

Right side affected PD

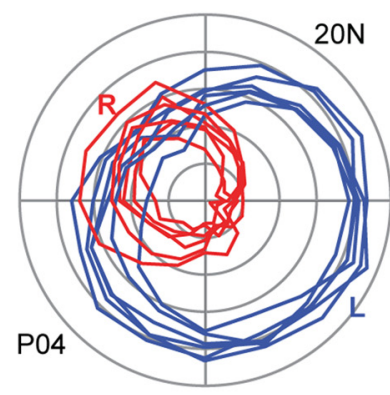

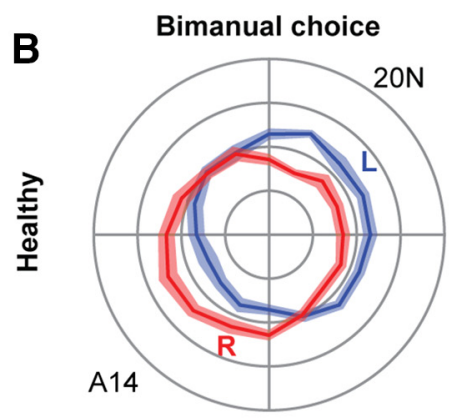
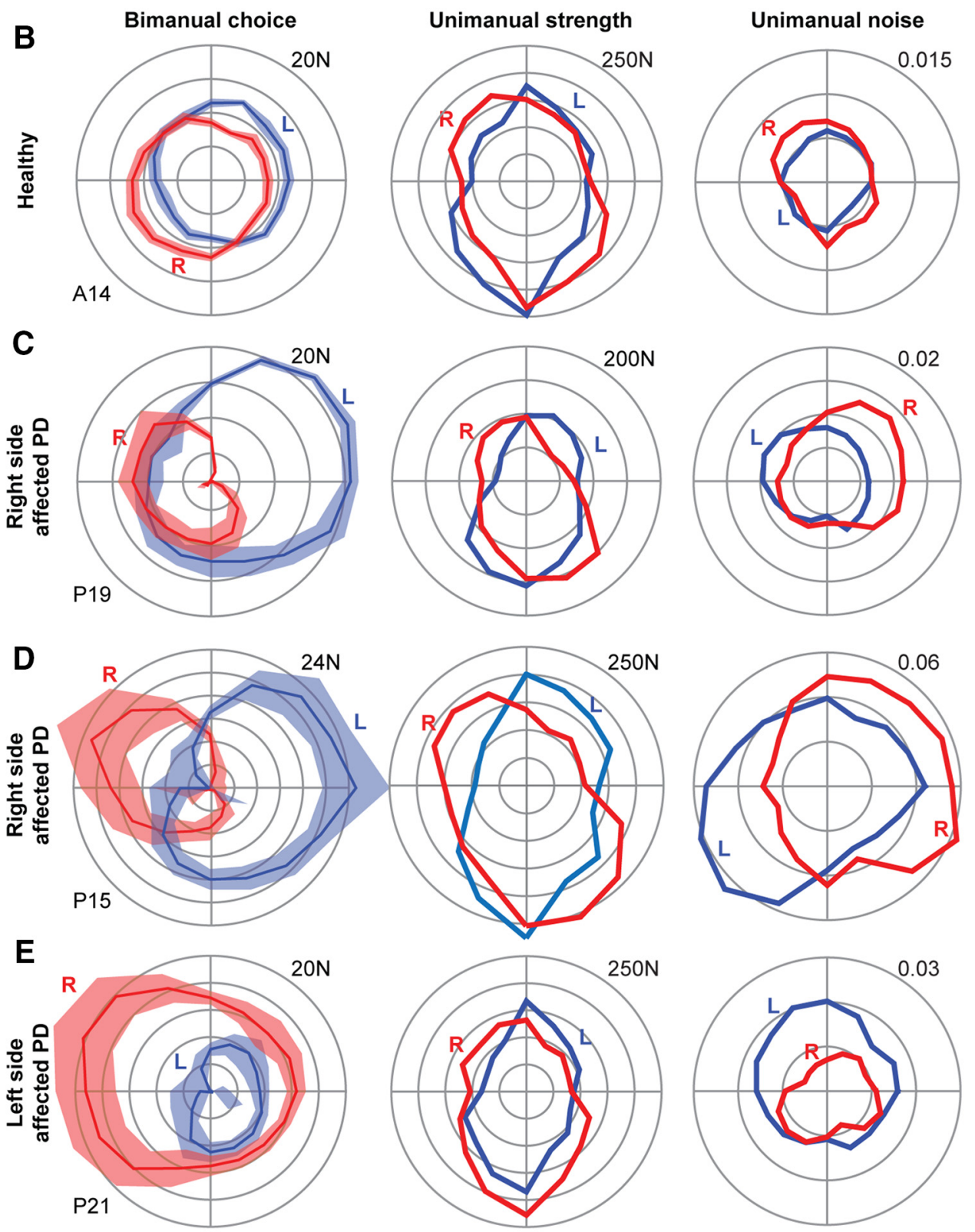

Figure 1. Experimental setup and data from representative subjects. $A$, The volunteer held a force transducer in each hand. The transducers were housed in the handles of two robotic arms. The task was to produce forces with the two arms so that the sum of the two forces would displace a cursor to a target at $20 \mathrm{~N}$. The targets were randomly selected from 16 directions and uniformly distributed around a circle. The task was isometric in that the robotic arms were programmed to produce a very stiff well that strongly resisted movement. $\boldsymbol{B}$, Data from a right-handed healthy volunteer (Subject A14). Left column displays the forces produced by each arm in the bimanual task. For example, when given a target force of $20 \mathrm{~N}$ at $+45^{\circ}$, the volunteer chose to produce $\sim 8 \mathrm{~N}$ by the right arm and $12 \mathrm{~N}$ by the left arm. Center column displays maximum voluntary force (MVF) produced by each arm in a unimanual test of strength. Right column displays signal-dependent noise for each arm in a unimanual test in which the arm produced forces at various levels and the SD of force was measured at each level. Signal-dependent noise is the slope of the SD of force as a function of mean force in each direction for each arm. The larger value implies that the SD of force grew faster as a function of mean force. $C$, Data from a right-handed, right-side-affected PD patient (Subject P19). D, Data from another right-handed, right-side-affected PD patient (Subject P15). E, Data from a right-handed, left-side-affected PD patient (Subject P21). Error bars (shaded region) indicate mean \pm 1 SD as measured across 10 trials of a single session. $\boldsymbol{F}$, Reproducibility of bimanual choices. Two right-handed PD patients, one right-side affected (Subject P04) and the other left-side affected (Subject P21), were measured repeatedly in the bimanual task over the course of 2 months. Each line represents the mean from a single session.

Each trial began with a $1 \mathrm{~cm}$ radius target placed at the center of the workspace (Fig. 1A). After $1 \mathrm{~s}$, the center target was removed and a goal target appeared in one of 16 locations equally spaced around a circle of radius $10 \mathrm{~cm}$. The objective was to produce a $20 \mathrm{~N}$ force vector along the direction of the target for $2 \mathrm{~s}$. The participant could accomplish this through any combination of forces in the two arms. The variables of interest were $\mathbf{f}_{L}$ and $\mathbf{f}_{R}$, the force produced by the left and right arms, respectively. Visual feedback of the cursor and target were available throughout the trial. After the cursor was maintained within the target region for $2 \mathrm{~s}$, the goal target disappeared and the center target reap- peared. After the cursor was brought back to the center target, a new goal target was displayed immediately.

The arms were supported in the horizontal plane by an air-sled and positioned below an opaque screen upon which a projector painted the targets. We enforced the same posture throughout the various experimental sessions by securing the shoulder to the chair and by placing a brace on each wrist. The shoulder and the elbow angles were $\sim 65^{\circ}$ and $60^{\circ}$, respectively. Target directions were presented in a pseudorandom order and each direction was repeated 10 times, resulting in 160 trials. 
Unimanual tasks. The force produced by each arm was a random variable that was affected by noise. We measured this noise independently for each arm in a unimanual task. The setup for the unimanual task was similar to the bimanual task in that a force on the transducer displaced a cursor. On each trial, a target was displayed, corresponding to 10,15 , or $20 \mathrm{~N}$ at a random direction distributed evenly at intervals of $22.5^{\circ}$ around a circle. Visual feedback was provided until the cursor moved inside the target and then eliminated thereafter in that trial. The objective was to maintain the target force for $10 \mathrm{~s}$. We measured the mean and SD of force during this period. To do so, force was digitally processed using a fifthorder Butterworth filter with a low-pass cutoff of $25 \mathrm{~Hz}$ and then detrended (Craigmile et al., 2005). Subjects performed two trials with each arm for each instructed force magnitude and direction for a total of 192 trials.

In this unimanual task, for each target direction $\theta$, the measured force was represented as a scalar quantity that was generated by the following noise model:

$$
\begin{aligned}
f_{R} & =u_{R}\left(1+k_{R}(\theta) \phi\right) \\
f_{L} & =u_{L}\left(1+k_{L}(\theta) \phi\right) \\
\phi & \sim N(0,1)
\end{aligned}
$$

In Equation 2, $u_{R}$ is the motor command to the right arm, $k_{R}(\theta)$ is the direction dependent slope of the signal-dependent noise, and $\phi$ is a scalar random variable with normal distribution, zero mean, and variance 1 . To estimate $m_{R}(\theta)$, for each direction $\theta$, we measured the SD of force at each target force and then determined the slope of the SD of force versus mean force on that trial using linear regression, constraining the intercept to zero. As a result, the unimanual variance measurements produced an estimate of signal-dependent noise in each of the 16 directions for each arm.

For each target direction, we also measured the maximum voluntary force that subjects could produce by each arm and labeled these measurements with the scalar functions $m_{R}(\theta)$ and $m_{L}(\theta)$. Participants were instructed to produce the highest possible force in each direction and the maximum value was recorded ( 32 trials, two trials for each direction). The robotic arms were not used in these measurements because the maximum voluntary forces exceed the capabilities of the robot motors. Rather, a force transducer was mounted at the location of each handle and held in place with bolts that secured the transducer to a rigid frame.

Experiment 1a: Subjective cost of force production. Two groups of volunteers participated in this experiment: a right-handed group of healthy volunteers ( $n=15$, age $57 \pm 8.6$ years, mean \pm SD, 5 females $)$ and a right-handed group of PD patients $(n=15$, age $58.6 \pm 6.6$ years, 6 females). The characteristics of the patients are provided in Table 1 . The patients had disease duration of $7.9 \pm 5.2$ years, were prescribed Levodopa or an equivalent medication dosage of $497 \pm 298 \mathrm{mg} / \mathrm{d}$, and had a motor Unified Parkinson's Disease Rating Scale (UPDRS) score of $17.0 \pm 6.0$. All patients in this experiment were right-side affected. The experiment began with the Edinburgh Handedness Survey (Oldfield, 1971). After completion of the survey, subjects performed the bimanual task, the unimanual noise task, and finally the unimanual maximum voluntary force task.

Experiment 1b: Reproducibility of the results. To assess the reproducibility of our results, we measured bimanual behavior in 2 patients repeatedly over the course of a 2 month period. A left-side-affected patient (P21) was tested on 7 separate days and a right-side-affected patient (P04) was tested on 6 separate days.

Experiment 2: Anodal stimulation of left M1. Current was delivered through the electrodes using a Phoresor II Auto device (Model PM850; IOMED) and lasted $25 \mathrm{~min}$. The experiment began with placement of the electrodes on the head. The electrodes were $25 \times 5 \mathrm{~cm}^{2}$ sponges soaked in saline. The motor cortex on each hemisphere was localized using the 10-20 EEG system, with one electrode placed on marker C3 (left hemisphere) and the other placed on marker C4 (right hemisphere).

We asked $n=10$ of the patients that had participated in Experiment 1 to return (Table 1 , age $59 \pm 7.1$ years, mean \pm SD, 4 females). The first 10 patients that had participated in Experiment 1 were recruited for Experiment 2 and the rest were recruited for Experiment 3. They had disease duration of $6.9 \pm 4.6$ years, were prescribed Levodopa or an equivalent medication dosage of $470 \pm 320 \mathrm{mg} / \mathrm{d}$, and had a motor UPDRS score of $15.7 \pm 4.9$. The experiment began with measurement of UPDRS, followed by the bimanual task, the unimanual noise task, and the unimanual MVF task. Next, we placed the electrodes on the head and applied $1 \mathrm{~mA}$ of anodal stimulation over the left M1 with the cathode placed over the right M1. Once stimulation began, we measured behavior in the bimanual task, the unimanual noise task, and the unimanual MVF task. Stimulation lasted $\sim 25 \mathrm{~min}$. The experiment ended with measurement of motor UPDRS.

Experiment 3: Cathodal stimulation of left M1. We recruited $n=10$ right-handed, right-side-affected PD patients (Table 1) to participate in this single-day experiment. The experiment began with measurement of motor UPDRS, followed by the bimanual task, the unimanual noise task, and the unimanual MVF task. Next, we placed the electrodes on the head and applied $2 \mathrm{~mA}$ of anodal stimulation over the right M1 with the cathode placed over the left M1. During stimulation, we measured behavior in the bimanual task, the unimanual noise task, and the unimanual MVF task. Stimulation lasted $\sim 25 \mathrm{~min}$. The experiment ended with measurement of motor UPDRS.

Experiment 4: Double-blind tDCS. We recruited $n=10$ right-handed PD patients (Table 1 ) to participate in a $3 \mathrm{~d}$ double-blind $\mathrm{tDCS}$ study. On each day, the volunteers received sham, anodal, or cathodal stimulation of the hemisphere contralateral to the affected side, randomly assigned. Neither the patient nor the neurologist who performed the clinical assessment (motor UPDRS) was aware of the pattern of stimulation. On each day, we began with placement of the electrodes and start of stimulation. We then measured behavior in the bimanual task. The procedures for each day ended with measurement of motor UPDRS by a neurologist blind to the form of stimulation.

Experiment 5: Effects of long-term stimulation. We recruited $n=8$ right-handed PD patients (Table 1) to participate in a $10 \mathrm{~d}$ single-blind experiment. On Day 1, the volunteers received no stimulation. On Days 2 and 3 , they received sham stimulation. On Days $4,5,6,7$, and 8 , they received real stimulation. Finally, on Days 9 and 10, they received no stimulation.

On Day 1, the experiment consisted of measurement of motor UPDRS, followed by measurement of behavior in the bimanual task, the unimanual noise task, and the unimanual MVF task. On Day 2, we performed sham stimulation. We placed the electrodes with anode over the M1 ipsilateral to the affected side and the cathode over the M1 contralateral to the affected side. Over a period of $30 \mathrm{~s}$, the current was increased from 0 to $2 \mathrm{~mA}$, held for $10 \mathrm{~s}$, and then decreased over a $30 \mathrm{~s}$ period back to zero. We then measured behavior in the bimanual task, the unimanual noise task, and the unimanual MVF task. The session ended with measurement of motor UPDRS. On Day 3, we repeated the sham stimulation, remeasured behavior in the bimanual task, and remeasured motor UPDRS. On Day 4, the participants received real stimulation. The protocol consisted of $2 \mathrm{~mA}$ of cathodal stimulation of the $\mathrm{M} 1$ contralateral to the affected side with the anode placed over the M1 ipsilateral to the affected side. We measured behavior in the bimanual task, the unimanual noise task, and the unimanual MVF task. On Days 5, 6, 7, and 8, we repeated the stimulation, remeasured behavior in the bimanual task, and remeasured the motor UPDRS. On Day 9, the session began with measurement of behavior in the bimanual task, the unimanual noise task, and the unimanual MVF task. The session ended with measurement of motor UPDRS. On Day 10, we remeasured behavior in the bimanual ask, and finally remeasured motor UPDRS. On average, the total length of this experiment was $28.0 \pm 3.9$ calendar days.

In addition to these objective measures, we also asked the patients to provide us with a subjective assessment of their disease symptoms. They filled out the PDQ-39 questionnaire (Peto et al., 1995) on Day 1 before start of the study and on Day 10 after completion of the study. This questionnaire provided a self-assessment of mobility, activities of daily living, emotional wellbeing, stigma, social support, cognition, communication, and bodily discomfort.

Mathematical modeling. To understand behavior in the bimanual task, we considered a cost that penalized motor commands based on strength of each arm and noise in each arm. The idea was that, for a given arm, 
force at a given direction would cost less if that arm was stronger than the other arm, but would cost more if that arm was more variable than the other arm.

In the model, we began with the requirement that the forces produced by the two arms must sum to the target force as follows:

$$
f^{*}=u_{L}+u_{R}
$$

In Equation $3, f^{*}$ is the target force $\left(20 \mathrm{~N}\right.$, a scalar quantity), and $u_{L}$ and $u_{R}$ are the forces produced by the right and left arm, also scalar quantities, respectively. Equation 3 represents an ill-posed problem for the brain: it is unclear how much force one should produce by each arm in order for the sum to equal the target. People's behavior, however, suggested a consistent and reproducible solution. To account for that behavior, we considered a cost that was composed of two terms, one that penalized noise, and one that penalized effort, as follows:

$$
J=\frac{1}{2 \operatorname{tr}[K(\theta)]} \mathbf{u}^{T} K(\theta) \mathbf{u}+\frac{1}{2 \operatorname{tr}[M(\theta)]} \mathbf{u}^{T} M(\theta) \mathbf{u}
$$

In the above equation, $t r$ is the trace operator and $\mathbf{u}=\left[\begin{array}{ll}\boldsymbol{u}_{L} & \boldsymbol{u}_{R}\end{array}\right]^{T}$ and $K(\theta)$ is a matrix that describes a quantity related to signal dependent noise for each arm as follows:

$$
K(\theta)=\left[\begin{array}{cc}
k_{L}^{2}(\theta) & 0 \\
0 & k_{R}^{2}(\theta)
\end{array}\right]
$$

$M(\theta)$ is a matrix that describes a quantity related to maximum voluntary force for each arm as follows:

$$
M(\theta)=\left[\begin{array}{cc}
m_{L}^{-2}(\theta) & 0 \\
0 & m_{R}^{-2}(\theta)
\end{array}\right]
$$

Note that, in this model, there are no free parameters and therefore nothing to fit: we measure MVF, which determines $\mathbf{u}$, and we measure signal-dependent noise, which determines $K(\theta)$.

The idea behind the cost function is as follows. For a given target direction, force for a given arm will cost less if that arm is stronger than the other arm. Similarly, force for a given arm will cost less if that arm has less noise than the other arm. The exact force that will be assigned to each arm will equally take into account these two costs.

The mathematical problem was to find the motor commands $\mathbf{u}$ that minimized the cost in Equation 4 given the constraint in Equation 3. We solved this problem using Lagrange multipliers, which produced a closed form solution as a function of the known quantities $K(\theta), M(\theta)$, and $f^{\prime}$ as follows:

$\mathbf{u}^{*}=\left(\frac{K(\theta)}{\operatorname{tr}[K(\theta)]}+\frac{M(\theta)}{\operatorname{tr}[M(\theta)]}\right)^{-1} \mathbf{p}^{T}\left(\mathbf{p}\left(\frac{K(\theta)}{\operatorname{tr}[K(\theta)]}\right.\right.$

$$
\left.\left.+\frac{M(\theta)}{\operatorname{tr}[M(\theta)]}\right)^{-1} \mathbf{p}^{T}\right)^{-1} f^{*}
$$

In Equation 7, $\mathbf{p}=\left[\begin{array}{ll}1 & 1\end{array}\right]$.

Despite having no free parameters, this model was able to make accurate predictions regarding behaviors of both healthy and PD subjects. However, analysis of the model errors suggested that, in PD, deviations between the model and the data could be due to the role of signaldependent noise. Therefore, we also considered a model with one free parameter, $q$, in which signal-dependent noise was penalized as follows:

$$
K(\theta)=\left[\begin{array}{cc}
k_{L}^{q}(\theta) & 0 \\
0 & k_{R}^{q}(\theta)
\end{array}\right]
$$

That is, rather than assuming that noise penalized motor commands as a squared variable (Eq. 5), we allowed it to be a free parameter that was fitted to the data. To find $q$, we minimized the sum of squared errors across directions $i=1, \ldots, 16$ between the observed and predicted motor commands $\sum_{i}\left(\mathbf{u}_{i}-\mathbf{u}_{i}^{*}\right)^{T}\left(\mathbf{u}_{i}-\mathbf{u}_{i}^{*}\right)$. We used bootstrapping to select subjects in each group, with replacement, and fitted the model to the group data. We then repeated the procedure to find the distribution associated with the unknown parameter.

Data analysis. In the bimanual task, we found that, when asked to produce a target force, each arm produced a force vector that was approximately parallel with the target vector (average absolute difference of $9.9 \pm 4.1^{\circ}$ for the healthy control group and $18.9 \pm 7.7^{\circ}$ for the PD group). For each target, we used as our proxy for force a scalar measure, the projection of the force vector produced by each arm onto the target vector.

Our data consisted of force measures taken from each arm along 16 directions. In the bimanual task, we used the fraction of the total force produced by a given arm as the measure along each direction and then computed the effect of direction and group using repeated-measures ANOVA (Mathematica 8; Wolfram). To summarize the data across directions, we computed a laterality index. The laterality index for the force produced by the right and left arms in the bimanual task was defined as follows:

$$
L=\frac{1}{16} \sum_{i=1}^{16} \frac{f_{R}^{(i)}-f_{L}^{(i)}}{f_{R}^{(i)}+f_{L}^{(i)}}
$$

In the above equation, a positive laterality index implies that the subject used their right arm more than left.

To estimate the subjective cost of force, for each direction of instructed force in the bimanual task we measured the force produced by each arm, and then found the ratio of the two forces, normalized by the strength of each arm in that direction. The direction-dependent subjective cost of force in the right arm was calculated as follows:

$$
C_{R}(\theta)=\frac{m_{L}^{-1}(\theta) f_{L}}{m_{R}^{-1}(\theta) f_{R}}
$$

Equation 10 implies that, if the subject chooses to produce a greater force with their right arm (normalized to strength), then the subject cost is lower for that arm than the left arm. The subjective cost of the left arm is simply the reciprocal of the subjective cost of the right arm.

We estimated noise properties of motor commands in two ways: in the time domain using signal-dependent noise (Eq. 2) and in the frequency domain using spectral analysis. To estimate noise characteristics in the frequency domain, we computed a power spectral density of force in the unimanual task for each instructed direction and magnitude. The force $f(t)$ that we measured in the unimanual noise task was transformed to produce an estimate of the power spectral density at frequencies ranging from 1 to $10 \mathrm{~Hz}$ as follows:

$$
P=\lim _{T \rightarrow \infty} \frac{1}{4 \pi T} \int_{-\infty}^{\infty} \int_{-\infty}^{\infty} \int_{-T}^{T} f(t) f(t+\tau) e^{-j w \tau} d t d \tau d w
$$

We used Welch's averaged modified periodogram method of spectral estimation with a window size of 256 samples with 128 overlapping samples between two adjacent windows. Because the force signal was sampled at $200 \mathrm{~Hz}$, this approximated a $0.7813 \mathrm{~Hz}$ frequency bin for each power spectral estimate. The Goertzel algorithm was used to estimate the power spectral density in any specific frequency range (Goertzel, 1958).

To compare data between two groups or within-subject changes, we first checked for normality of the data using the Shapiro-Wilks test. Only if the data were normally distributed did we continue on with a $t$ test. Otherwise, a location test with the Wilcoxon signed-rank test, a nonparametric statistical comparison, was used.

\section{Results}

In our isometric task, the participants were instructed to produce a target force by using both arms, but were free to select the force assigned to each arm. That is, they chose how much force to produce with each arm such that the sum matched an instructed magnitude and direction (Fig. $1 A$ ). For example, when a healthy, right-handed volunteer was instructed to produce a $20 \mathrm{~N}$ force 
(Fig. 1B, left column), he exhibited a preference for his right arm in some directions (e.g., $-135^{\circ}$ ) and a preference for his left arm in other directions (e.g., $+45^{\circ}$ ). However, in all directions, he shared the burden between the two arms. In contrast, when a right-handed, right-side-affected PD patient performed the same task, for some directions, he relied almost exclusively on his left arm (Fig. 1C, left column; clinical symptoms in Table 1). This was not because the right arm was weak or incapable of producing large forces, as illustrated by maximum voluntary forces produced by each arm (Fig. $1 C$, center column). Indeed, for $0^{\circ}$, the left and right arms of this patient were of comparable strength. However, when given a choice, the patient preferred to use his left arm to the near exclusion of his right arm. Importantly, this extreme bias toward the left arm was not present in all directions. For example, when asked to produce a force toward $+135^{\circ}$, the patient shared the burden with the two arms, behaving much like the healthy subject.

Another example of a right-handed, right-side-affected PD patient is shown in Figure $1 D$. Whereas, for most directions, the patient strongly preferred his left arm (e.g., $0^{\circ}$ ), there were also some directions for which he strongly preferred his right arm (e.g., $+135^{\circ}$ ) despite the fact that he was capable of producing large forces with both arms in all directions (Fig. $1 D$, center column).

Finally, consider a right-handed PD patient who has been affected on the nondominant side (Fig. $1 E$ ), a relatively rare condition (van der Hoorn et al., 2012). When asked to produce a force at $180^{\circ}$, she relied almost exclusively on her right arm. However, for other directions (e.g., $-90^{\circ}$ ), she shared the burden between the two arms.

These data represent preferences as measured in a single session. Were the patterns stable over time? We examined two of the patients on multiple sessions spaced over a 2 month period and have plotted the results in Figure $1 F$, where each line represents measurements from a single session. On each session, we computed a laterality index (Eq. 9). A positive laterality index indicated a preference for the right arm and a negative index indicated a preference for the left arm. We found that performances were remarkably stable: ANOVA on the laterality index measured each day found no effect of day in either of the patients (left-side-affected $F_{(1,5)}=0.06, p=0.81$; rightside-affected $\left.F_{(1,4)}=0.57, p=0.49\right)$. Therefore, it appeared that the patients consistently preferred to produce force with their less-affected arms, but only for certain directions.

To better understand the origin of these choices, we also measured the noise while the patients produced force with each arm. In a unimanual isometric task, the subjects maintained a prescribed level of force at a given direction without visual feedback and we measured the SD of the force that they produced as a function of mean of that force. The slope of this relationship, that is, the rate with which SD increased as a function of force magnitude, provided an estimate of signal-dependent noise. We computed signal-dependent noise for each direction of force production in each arm. The results are plotted in the right column of Figure 1, $B-E$. In general, it appeared that the direction for which the noise was smaller in an arm was usually the direction for which the patient showed a preference for that arm. We tested this conjecture in Experiment 1.

In summary, we observed that, when $\mathrm{PD}$ patients were asked to produce a target force through activation of both arms, they assigned most of the force to the less-affected arm, but this preference depended on the direction of the target force. For some directions, they shared the burden between the two arms, whereas for other directions, they assigned the burden nearly exclusively to the less-affected arm. Arm strength by itself could not account for the preferences, but there was some indication that the preferences were partly a reflection of signal-dependent noise in each arm. The noise was generally larger in the affected arm and the patients preferred to assign a greater force to the less noisy arm.

\section{Experiment 1: Subjective cost of force}

We recruited a group of right-handed, right-side-affected PD patients $(n=15)$ and an equal number of healthy, age-matched controls. In the bimanual task (Fig. $2 A$ ), healthy people exhibited a preference for use of the left arm for some directions (e.g., $+45^{\circ}$ ) and a preference for use of the right arm for other directions (e.g., $+135^{\circ}$ ). In $\mathrm{PD}$, for some directions, the force assigned to the two arms appeared comparable to healthy controls (e.g., $+135^{\circ}$, as shown in Fig. $2 A$ ); that is, for some directions, the PD patients shared the burden between the two arms normally. However, for other directions, these right-side-affected PD patients showed a marked preference for their left $\operatorname{arm}\left(\right.$ e.g., $\left.0^{\circ}\right)$.

To compare performance statistically, for each direction and each subject, we computed a laterality index, the difference between the force on the right and left arms, divided by the sum of the two forces. A repeated-measures ANOVA of the laterality index with the within-subject factor of direction and a betweensubject factor of group produced a significant effect of direction $\left(F_{(15,420)}=42, p<10^{-9}\right)$, a significant effect of group $\left(F_{(1,28)}=\right.$ $\left.25.3, p=2.5 \times 10^{-5}\right)$, and a significant direction by group interaction $\left(F_{(15,420)}=8.8, p<10^{-9}\right)$. This result implied that, whereas the contribution of each arm was a direction-dependent variable, across directions, the healthy subjects tended to use the two arms approximately equally (laterality index, averaged over all direction, right-subplot of Fig. $2 A$ ), but the right-side-affected $\mathrm{PD}$ group preferred to produce force with their left arm. Importantly, the data in Figure $2 A$ illustrate that the PD group did not have a global reluctance toward use of the affected side. Indeed, for some directions (second quadrant), the forces assigned to the two arms appeared normal. What might account for this direction-dependent behavior?

A simple hypothesis is that the preference for the left arm is due to weakness of the right arm (the more-affected arm). Therefore, we measured maximum voluntary force produced by each arm (Fig. 2B). The strengths of the two arms appeared normal in PD patients. A repeated-measures ANOVA of laterality index of maximum voluntary force with the within-subject factor of direction and a between-subject factor of group produced a significant effect of direction $\left(F_{(15,420)}=37.9, p<10^{-8}\right)$, but no effects of group $\left(F_{(1,28)}=0.006, p=0.94\right)$ and no interactions $\left(F_{(15,420)}=\right.$ $0.64, p=0.84)$. That is, whereas the strength of each arm was a direction-dependent variable, the relative strengths of the two arms were normal in PD patients (Fig. $2 B$ ). Therefore, strength by itself could not account for the choices that the patients made.

To compute a subjective cost of force for each arm in each direction, we used the forces that the subjects had chosen to assign in the bimanual task and weighted these forces by the inverse of maximum voluntary force in that direction (Eq. 10). The greater the subjective cost, the less force would be assigned to that arm in the bimanual task. We found that, for the healthy subjects, the subjective costs for the left and right arms were near unity, implying that, in all directions, force in one arm had a cost that was equal to the force on the other arm. However, in PD patients, the cost for the right arm was grossly exaggerated, but only for directions of force in the first and fourth quadrants. As a 
A
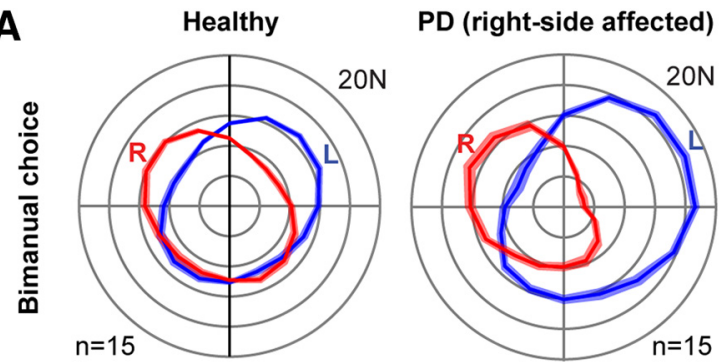

B
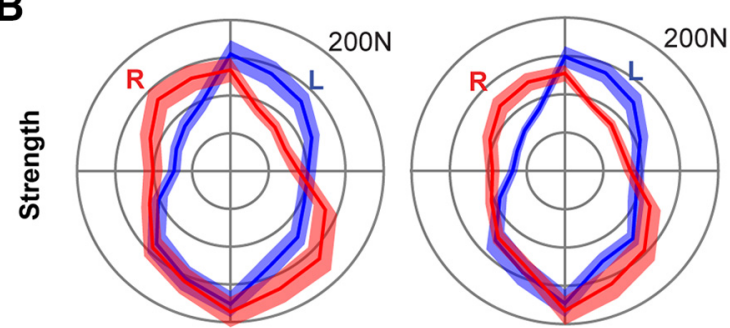

C
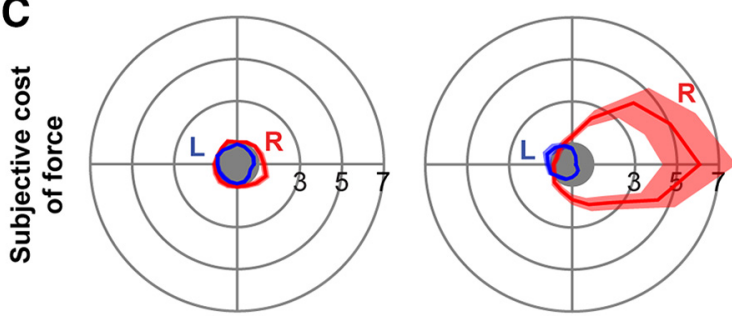
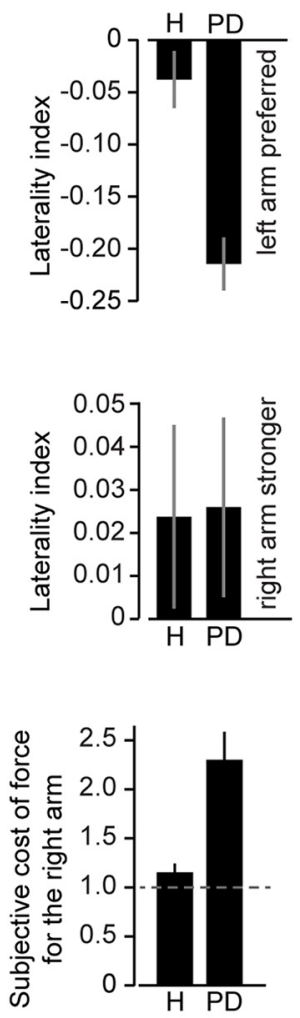

Figure 2. Performance of $n=15$ PD right-side-affected patients and $n=15$ age-matched healthy participants (Experiment 1). $\boldsymbol{A}$, Data from the bimanual task. In the bimanual task, the PD patients had a general preference for their left arm. The laterality index was computed for each subject via Equation 9 and had a theoretical range of -1.0 (complete preference for the left arm) to +1.0 (complete preference for the right arm). $\boldsymbol{B}$, Unimanual strength. The maximum voluntary force patterns appeared indistinguishable between the healthy and PD, with both groups exhibiting a slight strength advantage on the right side. C, Subjective cost of force, calculated via Equation 10, from unimanual strength and bimanual choices. In PD patients, the subjective cost of force production is much higher on the right side but only for certain directions. The data here are mean and SD estimated from bootstrapped population samples of each group. The right-most column is the mean subjective cost for the right arm (average of the data across all directions). The gray disk (unity) reflects equal subjective cost of force in the right and left arms. Error bars indicate SD.

result, on average, the subjective cost of force on the right arm in PD patients was approximately twice as large as in the healthy group (Fig. $2 C$, right; $p<0.005$ ).

\section{Noise as an implicit motor cost}

Why is the subjective cost of force in PD elevated in only certain directions? Let us consider the possibility that, in the right-sideaffected PD patient, the brain prefers to use the left arm in certain directions because force produced by the left arm is associated with less noise. In a unimanual task, we measured the SD of force as a function of mean force (Fig. $3 A$ ) for each direction. We found a significant within-subject effect of force magnitude in both the $\mathrm{PD}$ and the healthy groups $\left(\mathrm{PD}: F_{(2,36)}=14.75, p=0.0005\right.$; healthy: $\left.F_{(2,36)}=20.6, p=0.00006\right)$, demonstrating that SD of force increased with mean force. For each direction $\theta$ in each arm and for each subject, we found the slope of the relationship between SD of force and mean force. The result was an estimate of signal-dependent noise $k_{L}(\theta)$ and $k_{R}(\theta)$ for the left and right arms (Fig. $3 B$ ). A large signal-dependent noise implied that the SD of force grew rapidly as a function of mean force.

We then compared the signal-dependent noise in the right arm of the healthy and PD groups and found that, surprisingly, there were no significant differences in $k_{R}(\theta)$ between the two groups (repeated-measures ANOVA, no effect of group $F_{(1,28)}=$

$0.37, p=0.54$; no direction by group interaction $\left.F_{(15,420)}=0.37, p=0.98\right)$. There were also no significant difference in $k_{L}(\theta)$ between the two groups (repeatedmeasures ANOVA, no effect of group $F_{(1,28)}=0.24, p=0.63$; no direction by group interaction $F_{(15,420)}=1.36, p=$ $0.16)$. However, when we compared for each subject the signal-dependent noise in the right arm versus the left arm $k_{R}(\theta)-k_{L}(\theta)$, we found that whereas, in the healthy group, the two arms were equally noisy (laterality index near zero; Fig. $3 B$, right), in the right-side-affected $\mathrm{PD}$ group, the right arm was noisier than the left $\operatorname{arm}\left(F_{(1,28)}=6.5, p=0.016\right)$. Therefore, signal-dependent noise on the affected side of the PD patient was not greater than normal. Rather, unlike healthy age-matched controls, the noise was lateralized.

Can the noise patterns in PD patients provide an explanation for the choices that they made in the bimanual task? Consider direction $135^{\circ}$ (Fig. $2 A$ ). For this direction, the patients shared the burden with the two arms, producing forces that were similar to healthy controls (greater for the right arm than left). For this direction, the noises in the left and right arms of PD patients were similar, but the strength of the right arm was greater. The choice made by the patients appeared to be consistent with the strength and noise in their arms. Next, consider direction $0^{\circ}$. For this direction, the PD patients relied much more on their left arm than did the healthy controls. The strength of the two arms was similar for the two groups, but, for the PD group, the noise on their right side was greater than their left. The choice made by the patients again appears consistent with the strength and noise in their arms.

To account for the direction-dependent choices, we considered a motor cost that penalized force in each arm based on its strength as well as noise (Eq. 4). The arm that was stronger was assigned a greater burden (i.e., force would cost less), whereas the arm that was noisier was penalized and assigned a smaller burden (i.e., force would cost more). In this cost function, there was nothing to fit; that is, there were no free parameters and the model had to explain the direction-dependent choices in the bimanual task from only the subject-specific unimanual measures of strength and noise. Remarkably, we found that this simple cost was able to account for the general patterns of the data in both the healthy and PD populations. In the PD group, the populationaveraged unimanual measures of strength and noise accounted for the direction-dependent bimanual choices with a correlation of $r=0.940\left(p<10^{-6}\right)$. Similarly, in the healthy group, the population-averaged unimanual measures accounted for the bimanual choices with a correlation of $r=0.953\left(p<10^{-6}\right)$.

For each subject, we used their unimanual strength and noise and computed the model's predictions regarding bimanual choice, resulting in the distribution of forces shown in Figure 3C. The filled region in this figure represents the measured data and 
the line segments represent the predicted data. For the healthy subjects, we found that the predicted and measured data matched well in all directions. For the PD subjects, however, the predicted and measured data diverged in a specific region: quadrants 1 and 4 . That is, the cost that explained the behavior of healthy subjects underestimated the preferences of the PD patients specifically in directions for which noise in the arms of the PD patients was lateralized (quadrants 1 and 4).

We therefore considered a oneparameter cost function (Eq. 8) in which noise could be penalized by a power $q$ that could be other than 2 . We found that the best estimate for this parameter was $q=$ $4.6 \pm 0.6$ (mean $\pm \mathrm{SD}$ ) for the PD group, significantly higher than in control $(p<$ $0.0001)$. The addition of this one parameter allowed the cost function to fit the PD choices in all quadrants (Fig. 2D), reducing the mean squared error between model and data by $38 \%$ compared with the no-parameter model. This larger power for noise in PD patients implied that, when the noise in the two arms differed by a given amount, that difference resulted in a larger subjective cost of force in PD than in the controls. That is, the choices in PD patients suggested that they were more sensitive to the differences in noise in their arms than healthy controls.

In summary, the subjective cost of force production was elevated in $\mathrm{PD}$, but in only certain directions. Surprisingly, signal-dependent noise on the affected side was not higher in PD patients compared with controls. Rather, in righthanded, right-side-affected PD patients, the right side exhibited greater noise than the left side, a feature that was not present in controls. Therefore, signal-dependent noise was lateralized in PD patients, but notinage-matchedcontrols. Thedirectiondependent choices that the patients made in assigning force to each arm were accounted for with a cost that penalized motor commands to each arm based on both the strength and noise of that arm. This cost was skewed in PD patients such that it overpenalized noise differences between the two arms compared with controls. The subjective cost of force production was larger than normal in PD patients, but only in directions for which noise was lateralized.

\section{Experiment 2: Anodal stimulation produced no significant effects}

Was there a causal relationship between unimanual noise and the subjective cost of force? To answer this question, we looked for ways to alter bimanual choices. In our earlier work, we had ob-
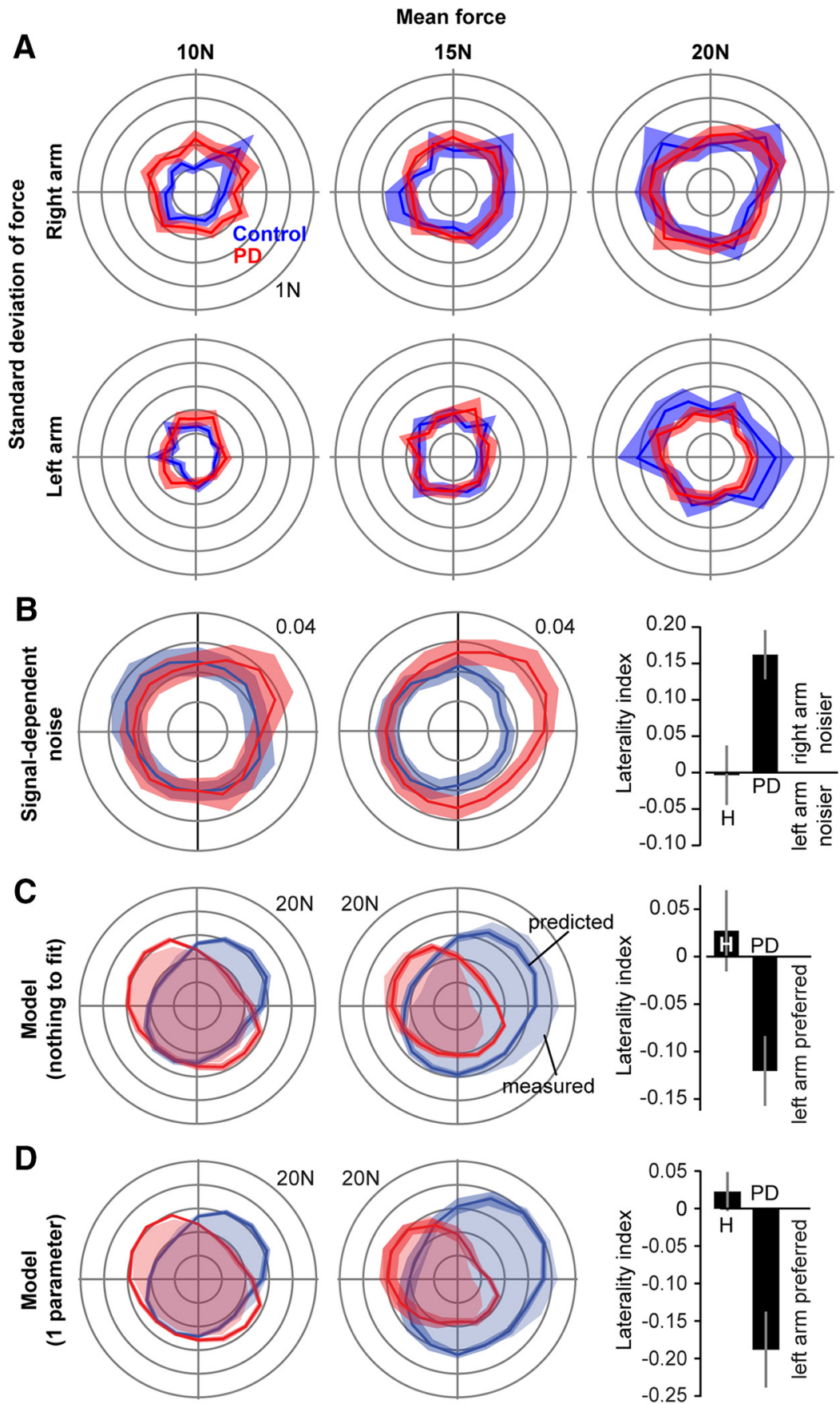

Figure 3. Unimanual signal-dependent noise patterns in PD. $A$, Noise as a function of force magnitude. On each trial, a target force was displayed $(10,15$, or $20 \mathrm{~N})$ at a random direction and the subject was asked to produce that force unimanually. Once the desired force level was reached, visual feedback was removed and the SD of the force was measured. The plot shows the measured SD at each target force for $n=15 \mathrm{PD}$ patients and $n=15$ healthy control subjects (Experiment 1). The slope of the relationship between SD and mean force was computed for each subject, each direction, and each arm, resulting in the signal-dependent noise variables $k_{R}(\theta)$ and $k_{L}(\theta)$. $\boldsymbol{B}$, Unimanual signal-dependent noise in PD patients was direction dependent and lateralized and generally higher in the right arm than the left. $\boldsymbol{C}$, Model of behavior with no free parameters. For each subject, the unimanual strength and signal-dependent noise were used to predict the contribution of each arm during bimanual control (Eq. 7). Error bars indicate the between-subject SEM. The filled region represents the measured data and the line segments are the predicted data. The laterality index on the right column is from the predicted data. D, Model of bimanual control with one parameter to fit (Eq. 8), allowing for nonquadratic penalty for noise. The filled region represents the measured data and the line segments are the fitted data. Error bars indicate SD as estimated from bootstrapping.

served that, in healthy people, noninvasive bilateral tDCS stimulation over the motor cortex altered choices in the bimanual task (Salimpour and Shadmehr, 2014). In particular, in right-handed young people, simultaneous anodal stimulation over the left M1 


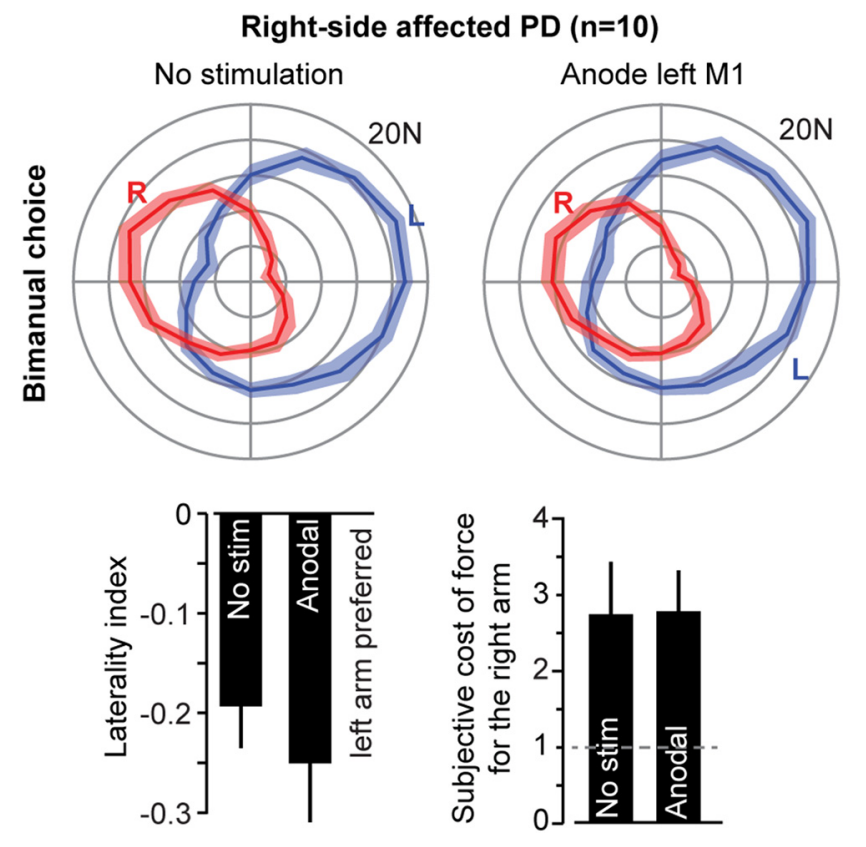

Figure 4. Effects of anodal stimulation of the left motor cortex. Shown are data for a session without stimulation and a session with $1 \mathrm{~mA}$ of anodal stimulation of the left M1 (cathode placed on the right M1). There were no significant effects of stimulation on bimanual choices or subjective cost of force. Error bars indicate SEM.

and cathodal stimulation over the right $\mathrm{M} 1$ increased the willingness of healthy subjects to assign force to their dominant right arm. This suggested that we might be able to increase the willingness of right-side-affected PD patients to use their right arm by exciting the left M1 while simultaneously inhibiting the right M1. We therefore recruited $n=10$ right-side-affected PD patients (Table 1, Experiment 2) and quantified their bimanual behavior with and without anodal stimulation of left M1 (cathode placed on right $\mathrm{M} 1$ ). We were surprised to find that, despite the stimulation, the forces that the PD patients assigned to each arm remained unchanged (Fig. 4, laterality index, within-subject comparison of stimulation with no stimulation, $t_{(9)}=1.4, p=$ $0.20)$.

\section{Experiment 3: Cathodal stimulation reduced subjective cost of force}

Next, we examined $n=10$ right-side-affected PD patients but applied cathodal stimulation to the left M1. The force patterns that the subjects produced before stimulation, during stimulation, and the within-subject change are plotted in Figure $5 \mathrm{~A}$. With cathodal stimulation we observed clear changes in preferences, demonstrating an increase in willingness to assign force to the affected side (right arm). Importantly, the changes were direction dependent: there appeared to be smaller changes in the first quadrant and larger changes in the second and third quadrants. ANOVA of change in force in right and left arms with direction as the repeated measure found a significant effect of $\operatorname{arm}\left(F_{(1,18)}=27.9, p=5 \times 10^{-5}\right)$ and a significant arm by direction interaction $\left(F_{(15,270)}=3.23, p=6 \times 10^{-5}\right)$. A post hoc within-subject comparison of laterality index confirmed that stimulation resulted in an increased willingness to produce force with the affected arm (laterality index; Fig. $5 A, t_{(9)}=3.73, p<$ $0.005)$. Indeed, cathodal stimulation produced significant improvements in the laterality index compared with anodal stimu- lation (Fig. 5E, Experiment 2 vs Experiment 3, $t_{(9)}=-3.66, p=$ 0.0018).

Did the stimulation affect signal-dependent noise? We found reductions in noise in the right arm but increases in the left arm (Fig. 5B). However, these changes were direction dependent: there appeared to be smaller changes in the first quadrant and larger changes in the second and third quadrant. ANOVA of change in noise in right and left arms with direction as the repeated measure found a significant effect of $\operatorname{arm}\left(F_{(1,18)}=6.54\right.$, $p=0.019)$ and a significant direction by arm interaction $\left(F_{(15,270)}=\right.$ 2.87, $\left.p=3 \times 10^{-4}\right)$. A post hoc within-subject comparison of laterality index for noise confirmed that stimulation had reduced the laterality of noise (Fig. $5 B$, laterality index; $t_{(9)}=2.98, p=$ 0.015). In fact, the laterality of noise had improved in 8 of 10 patients. Critically, the direction-dependent changes in unimanual noise were correlated with the direction-dependent changes in bimanual behavior. In directions for which noise in the left arm had increased, the force assigned to the left arm had decreased (group data, change in left arm noise and change in left arm force for each direction, $r=-0.59, p=0.015)$.

In contrast, stimulation produced no significant changes in unimanual strength (Fig. 5C). ANOVA of change in strength in the right and left arms with direction as the repeated measure found no main effects and no interactions.

We computed the subjective cost of force for the right arm (Eq. 10) and found that the stimulation had reduced this cost significantly (Fig. 5D, F; within-subject change in mean subjective cost of force for the right arm, $\left.t_{(9)}=-3.68, p=0.005\right)$. We also assessed clinical symptoms using the motor component of the Movement Disorder Society (MDS) UPDRS both before and after the stimulation (Fig. 5G) and observed a $25.4 \pm 7.7 \%$ reduction in the clinical scores (mean $\pm \mathrm{SD}$, signed-rank test, $p=$ 0.0058).

In summary, we observed that, in right-side-affected PD patients, $1 \mathrm{~mA}$ of anodal stimulation over the left $\mathrm{M} 1$ produced no significant changes in subjective cost of force. In contrast, $2 \mathrm{~mA}$ of cathodal stimulation over the left M1 significantly reduced the subjective cost of force for the right arm and also improved clinical symptoms. The improvement in bimanual behavior was direction dependent and coincided with direction-dependent changes in noise.

\section{Effect of repeated exposure to the task}

It is possible that some of the changes that we had seen in Experiment 3 were due to repetition of the task and not to stimulation per se. To quantify the potential effects of task repetition, we considered data from $n=13$ patients who had been tested on 2 separate days, both days in a no-stimulation scenario (nostimulation parts of Experiments 1, 2, and 3; Table 1). The force patterns that the subjects produced in these $2 \mathrm{~d}$, as well as the within-subject changes, are shown in Figure 6. The performances remained stable with repeated measurement (within-subject change in the laterality index, $t_{(12)}=0.964, p=0.35$ ). This result, combined with the fact that $1 \mathrm{~mA}$ of anodal stimulation produced little or no change in performance, gave an indication that the positive effects regarding cathodal stimulation may have been specific to the form of stimulation. To check for this directly, we performed a double-blind study.

\section{Experiment 4: Double-blind tDCS}

To test more rigorously the efficacy of the stimulation patterns, we performed a double-blind study in which we recruited $n=10$ patients and examined them on 3 separate days. On each day, the 
A

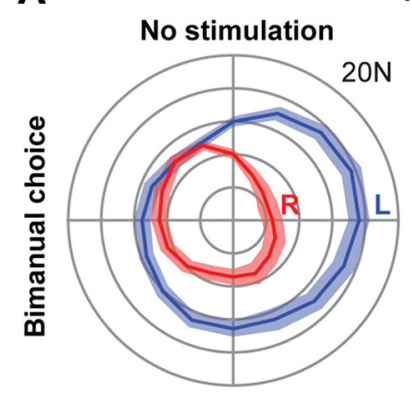

B

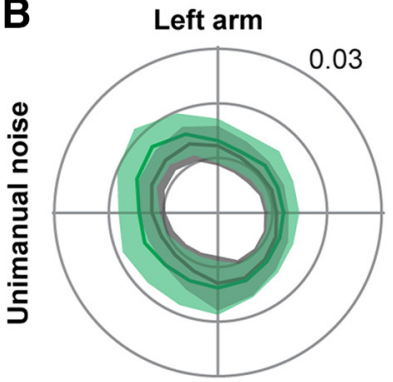

C
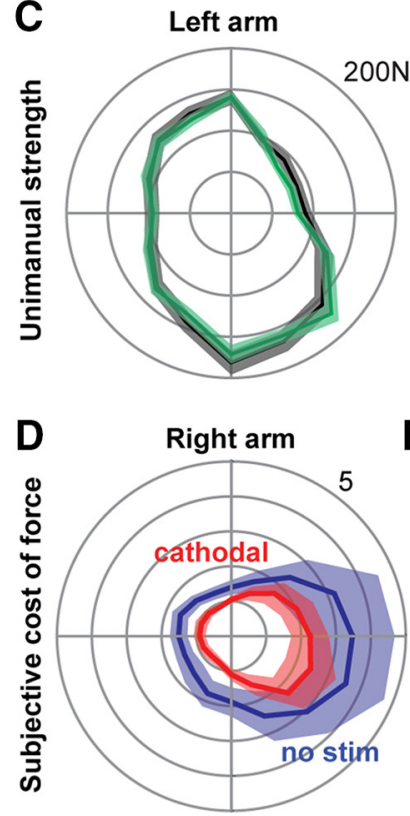

Right-side affected PD ( $n=10)$

Cathode left M1
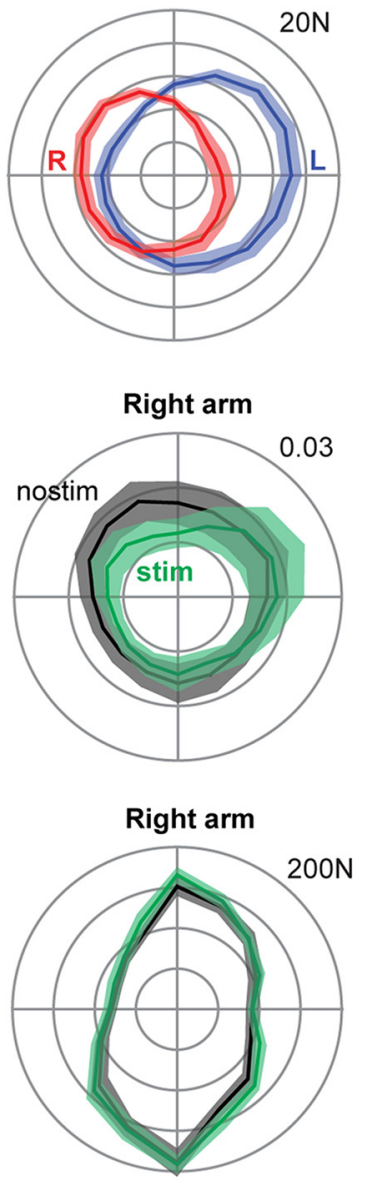

E

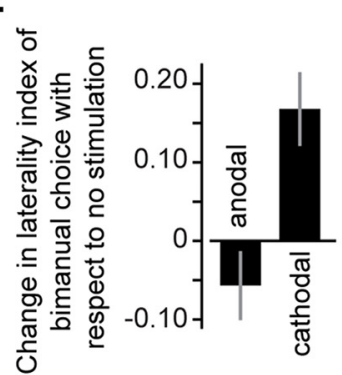

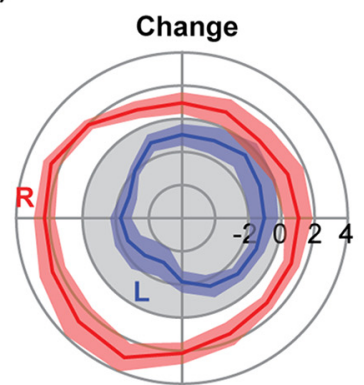

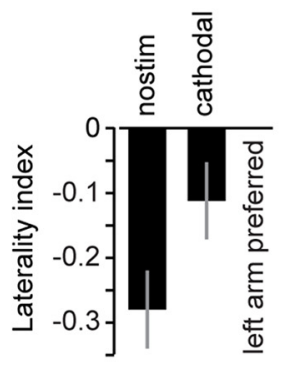

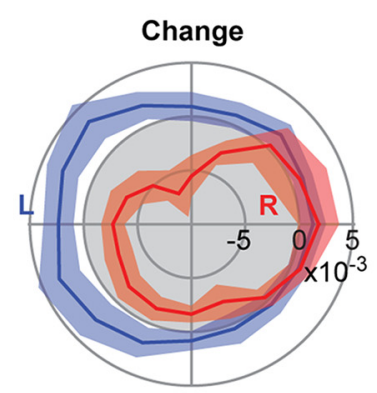
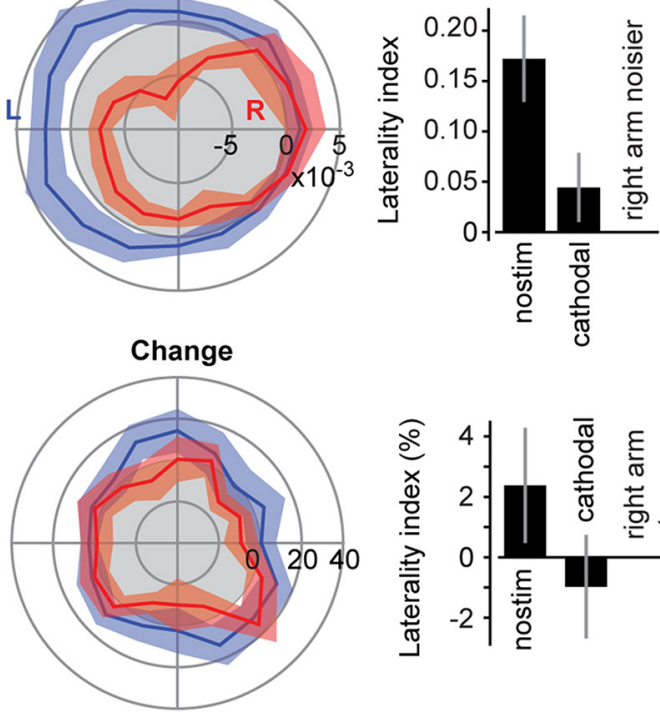

$\mathbf{F}$

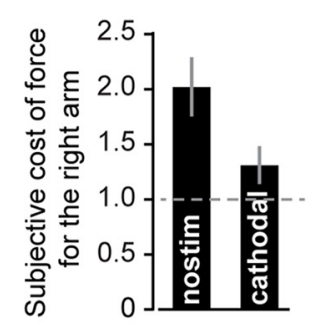

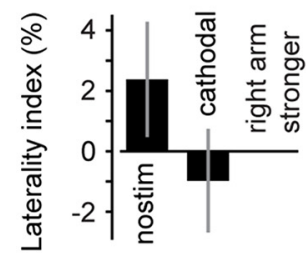

G

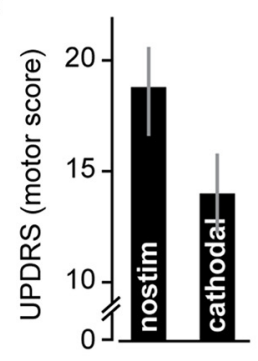

Figure 5. Effects of cathodal stimulation of the left motor cortex in a right-sided-affected PD patient. $A$, Behavior in the bimanual task without stimulation and with $2 \mathrm{~mA}$ of cathodal stimulation of the left M1 (anode placed on the right M1). The column labeled "change" represents within-subject difference between stimulation and no stimulation. The gray region indicates negative numbers. The data show that the willingness to exert force with the affected arm has increased. Rightmost column is the laterality index with and without stimulation. Error bars indicate SEM. B, Unimanual noise in each arm with and without stimulation. The column labeled "change" represents the within-subject difference between stimulation and no stimulation. Noise in the affected arm decreased, whereas noise in the left arm increased. Note that the directions for which the noise in the arm changed tend to correspond to directions for which bimanual behavior changed. Error bars indicateSEM. C, Unimanual strength (maximum voluntary force, MVF). Error bars indicate SEM. Stimulation had no significant effect on strength. D, Subjective cost of force for the right arm. $\boldsymbol{E}$, Change in the laterality index of bimanual choices after anodal stimulation (Experiment 2) and cathodal stimulation (Experiment 3). Cathodal stimulation significantly increased the willingness to exert force with the affected arm. $\boldsymbol{F}$, Mean subjective cost of force for the right arm averaged over all directions for each subject. The dashed line (unity) reflects equal subjective cost of force in the right and left arms. Error bars indicate SEM. Cathodal stimulation reduced the subjective cost of force for the right arm. G, Motor UPDRS in the no-stimulation and cathodal stimulation conditions. Error bars indicate SEM.

subject received sham stimulation, cathodal stimulation, or anodal stimulation over the motor cortex contralateral to the affected side. The order of stimulation was randomized. Importantly, neither the patient nor the clinician performing the clinical assessment was aware of the patterns of stimulation.

The force patterns that the subjects produced with sham, anodal, and cathodal stimulation are plotted in Figure 7A. Confirming our earlier findings, anodal stimulation produced no sig- nificant changes in the patterns of force production (Fig. $7 B$, laterality index, within-subject change with respect to sham, $\left.t_{(9)}=0.73, p=0.49\right)$. However, cathodal stimulation produced a clear increase in the willingness to assign force to the affected side: the laterality index declined significantly with cathodal stimulation compared with anodal stimulation (Fig. $7 B$, within-subject change, $\left.t_{(9)}=2.84, p=0.019\right)$ and compared with sham stimulation (within-subject change, $t_{(9)}=6.61, p=0.0001$ ). 


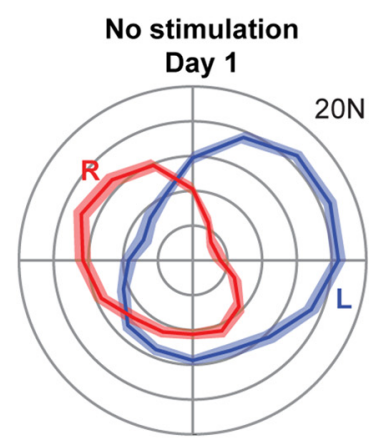

Right-side affected PD ( $n=13)$
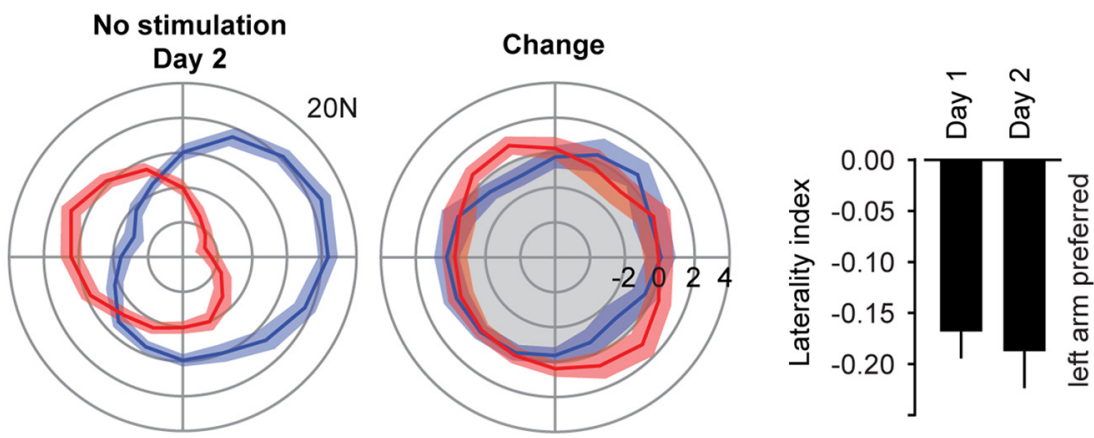

Figure 6. Effects of task repetition on performance. Data are from $n=13$ patients who were tested on 2 separate days, both days in a no-stimulation scenario (no-stimulation parts of Experiments 1,2, and 3; Table 1). The force patterns that the subjects produced in the bimanual task, as well as the within-subject changes, are shown (the gray region in the "change" figure indicates negative numbers; units are in Newtons). Rightmost column is the laterality index on each day. Error bars indicate SEM.

A

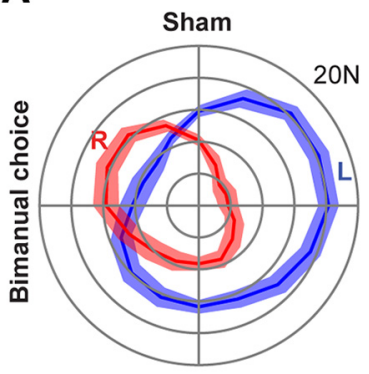

Double blind study $(n=10)$
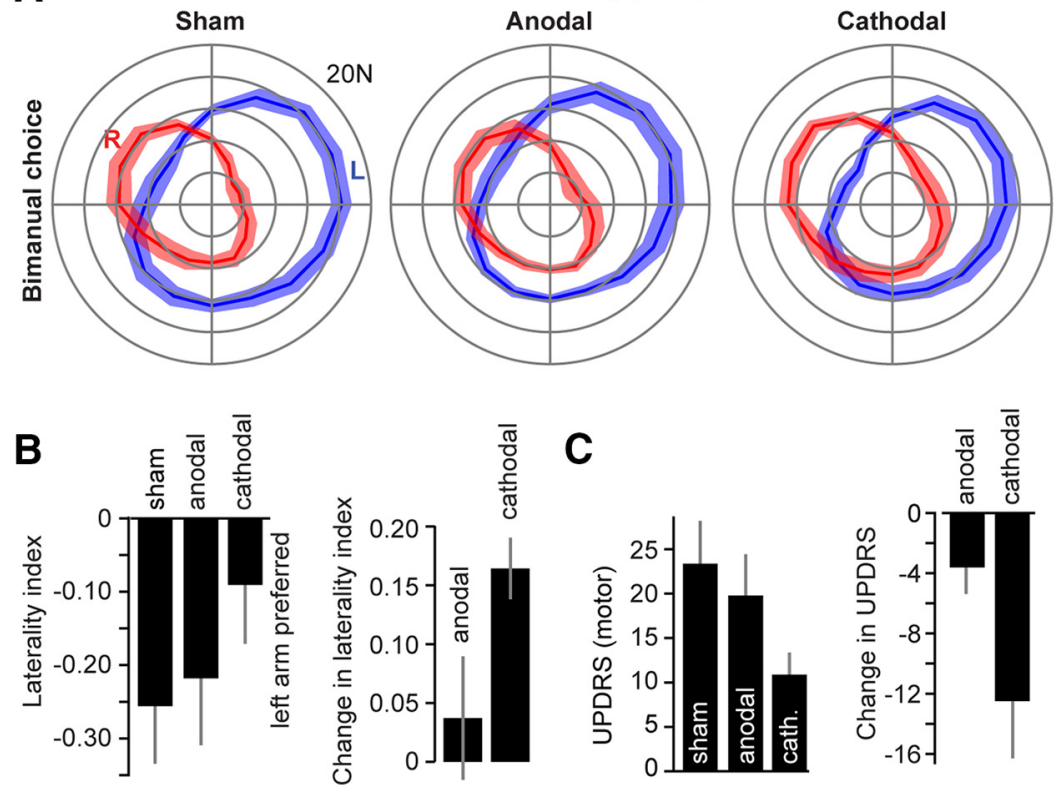

Figure 7. A double-blind study of $n=10$ patients who received sham, anodal, or cathodal stimulation randomly on separate days. $\boldsymbol{A}$, Bimanual choices. Cathodal stimulation appeared to increase the willingness to assign force to the affected right side. $\boldsymbol{B}$, Laterality index with sham, anodal, and cathodal stimulation. More negative numbers indicate preference for the left side. Change in laterality index is a within-subject measure of anodal or cathodal stimulation with respect to sham. Positive numbers indicate an increase in the willingness to assign force to the affected right side. C, Motor UPDRS. Change in UPDRS is a within-subject measure of anodal or cathodal stimulation with respect to sham. Larger negative numbers indicate a greater reduction in motor symptoms. Error bars indicate SEM.

The clinical symptoms, as measured via MDS-UPDRS, showed a trend toward significant changes after anodal stimulation (within-subject change with respect to sham, $t_{(9)}=-2.14$, $p=0.061)$. However, we observed a significant reduction in MDS-UPDRS after cathodal stimulation, both as measured with respect to anodal stimulation (within-subject change, $t_{(9)}=$ $-2.4, p=0.039$ ) and with respect to sham stimulation (withinsubject change, $\left.t_{(9)}=-3.39, p=0.008\right)$.

In summary, the improvements observed in behavioral measures and clinical symptoms appeared significantly larger with cathodal stimulation.

\section{Experiment 5: Effects of long-term stimulation}

We recruited $n=8$ patients for a $10 \mathrm{~d}$ study in which we measured performance without stimulation, with sham stimulation,

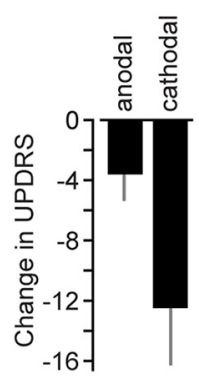

and with cathodal stimulation. Data acquired from a right-handed, right-sideaffected patient and a right-handed, leftside-affected patient are shown in Figure $8 A$. To assess the effects of stimulation, we compared within-subject change in performance on real stimulation days (compared with no stimulation) with change in performance on sham stimulation days. For laterality index, we found significantly larger improvements with real stimulation $\left(t_{(7)}=2.47, p<0.05\right)$. For subjective cost of force on the affected arm, we found significantly larger reductions with real stimulation $\left(t_{(7)}=-2.44, p<0.05\right)$. Furthermore, we observed that stimulation produced a change in unimanual noise, shifting the laterality index so that the affected side became less noisy compared with the unaffected side (Fig. $8 E$, withinsubject change in noise laterality index on stimulation days compared with average of sham and nonstimulation days, $t_{(7)}=$ $-2.43, p<0.05$; note that we measured noise on only one of the stimulation days). In contrast, stimulation produced no changes in unimanual strength (comparison of MVF on stimulation day compared with average of sham and no stimulation, $\left.t_{(7)}=-0.021, p=0.98\right)$.

On each day of the experiment, we also quantified clinical symptoms using MDS-UPDRS (Fig. 8D). We compared the within-subject effect induced by sham versus the effect induced by cathodal stimulation and found a significant effect of stimulation $\left(t_{(7)}=-6.84, p=0.0002\right)$. Finally, in addition to these objective measures, the patients provided us with a subjective assessment of their disease symptoms. They completed the PDQ-39 questionnaire on Day 1 before start of the study and on Day 10 after completion of the study, providing a selfassessment of mobility, activities of daily living, etc. We found a significant improvement in this measure of quality of life (within-subject comparison, $t_{(7)}=-5.06, p<0.002$ ).

\section{Effects of tDCS on noise}

How does tDCS alter the subjective cost of force? We have suggested that this effect may be due to changes that tDCS 
makes to unimanual signal-dependent noise, which itself may be due to an improved ability to recruit cortical neurons. Our estimate of noise thus far has quantified noise properties of force in the time domain. This approach misses the fact that noise is a frequency-dependent variable and that tremor may be higher in PD at specific frequencies. Therefore, we also analyzed unimanual force in the frequency domain and quantified the effects of cortical stimulation.

We computed the power spectrum of force as a function of frequency in the unimanual task for each direction and force magnitude and then, for each subject and each direction, averaged the data across various force magnitudes. For the volunteers who participated in Experiment 1, the integral of the resulting power spectrum is plotted as a function of direction in Figure $9 A$ and the power spectrum is plotted as a function of frequency is Figure $9 B$. We found that, in general, noise power dropped with increased frequency (repeated-measures ANOVA, $p<10^{-10}$ for the left arm, $p<10^{-10}$ for the right arm). Importantly, the noise power was higher in PD patients than in controls, especially for the right arm (repeatedmeasures ANOVA, $F_{(1,28)}=17, p<$ 0.0004 ) and particularly at higher frequencies. In addition, in this right-sideaffected group of patients, the noise power was lateralized (higher on the right side than left side), something that was not present in healthy controls (PD group, $t_{(14)}=4.8, p<0.0003$; control group, $p>0.5$ ).

We found that noise power was direction dependent (Fig. 9A). The direction dependency of noise correlated with the direction-dependent subjective cost of force in PD patients (group-averaged data, correlation between the integral of noise power spectrum in each arm and subjective cost of force in that arm, $r=$ $0.65, p=0.0048$ ).

Application of $2 \mathrm{~mA}$ of cathodal stimulation over the left M1 (Experiment 3 ) altered the noise spectrum broadly, differentially affecting the two arms (Fig. 9C). Repeated-measures ANOVA on the within-subject change in noise power produced no effect of frequency $\left(F_{(9,162)}=2.47, p=0.12\right)$, but a significant effect of $\operatorname{arm}\left(F_{(1,18)}=5.6, p=0.03\right)$. That is, cathodal tDCS altered noise power across the entire frequency spectrum, decreasing it on the right side and increasing it on the left side.

\section{Bilateral stimulation of the motor cortex}

Earlier experiments that had used a unilateral montage with the anode over the affected M1 had reported little or no effects in medicated PD patients (Benninger et al., 2010; Verheyden et al., 2013). Rather than unilaterally stimulate the more-affected M1, we bilaterally stimulated both cortical hemispheres. We selected
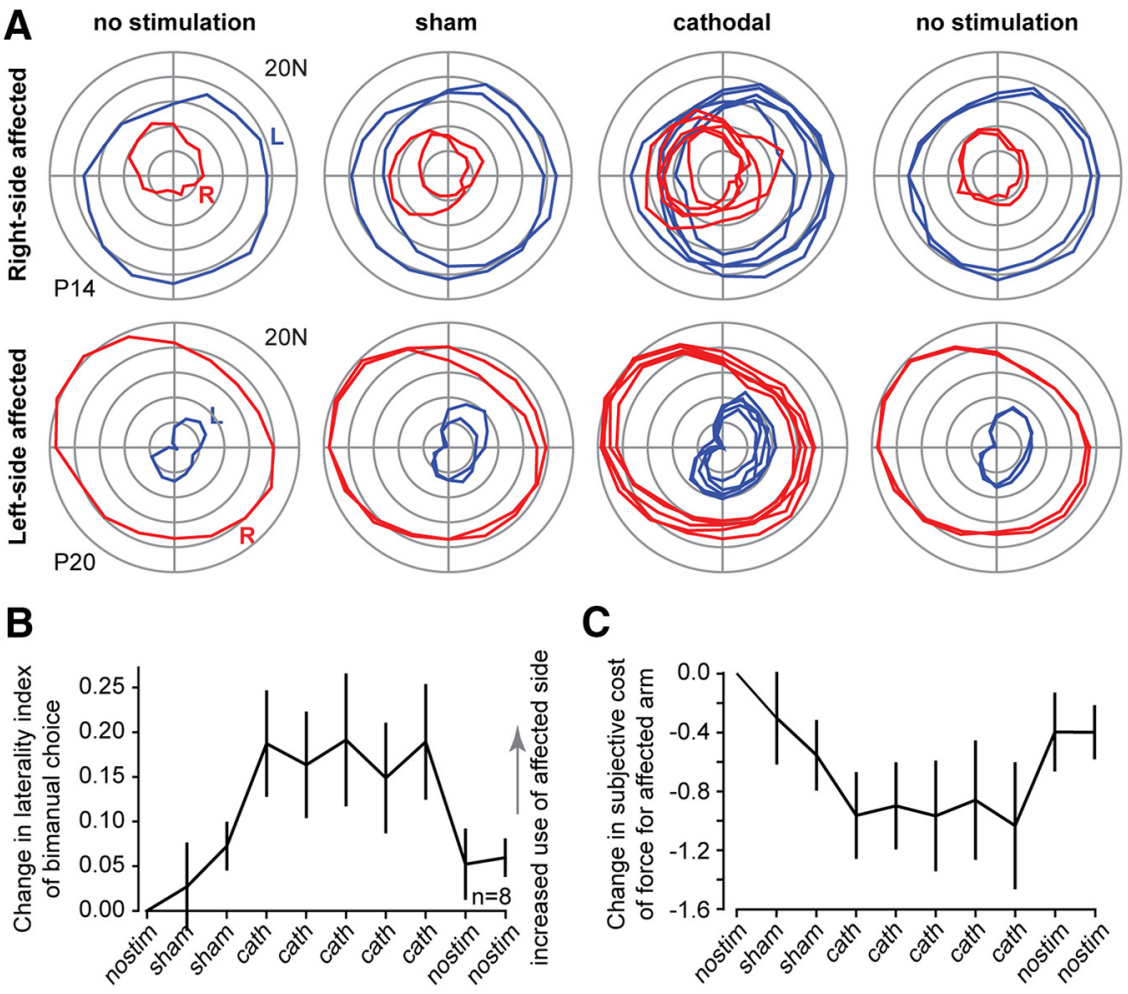

C

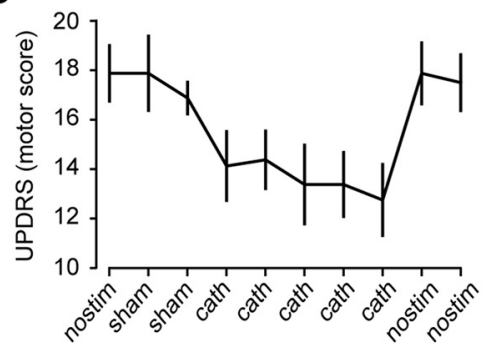

E

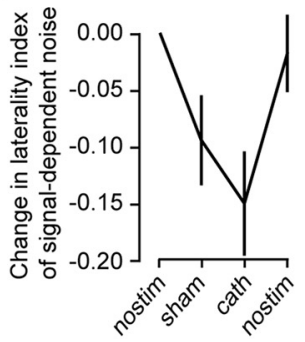

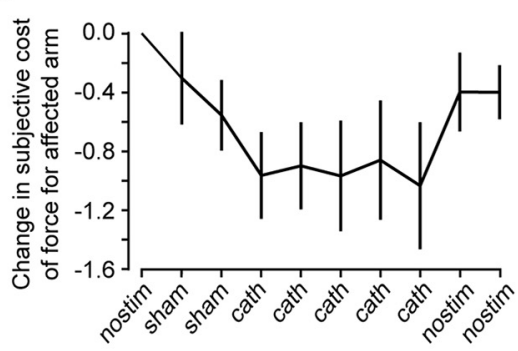

$\mathbf{F}$

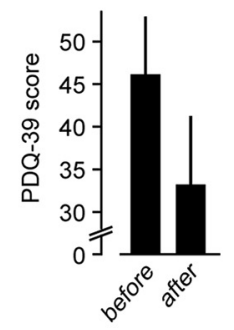

Figure 8. The $10 \mathrm{~d}$ repeated stimulation trial. We used cathodal stimulation of the motor cortex contralateral to the affected side in $n=8$ patients. The experiment began with a no-stimulation session, then two sessions of sham stimulation, then five sessions of cathodal stimulation, and finally two sessions of no stimulation. $\boldsymbol{A}$, Bimanual choices of two representative patients 列 index of unimanual noise. Noise measurements were taken only once on each specified session. Larger negative numbers indicate a reduction of noise in the affected side with respect to the unaffected side. $\boldsymbol{F}, \mathrm{PDQ}$-39 questionnaire scores before the start of the trial and after completion of the trial. Error bars indicate SEM.

this pattern because we hypothesized that unimanual noise was critical to effort costs and that our effect size might be largest if we could reduce the noise on the more-affected side while simultaneously increasing it on the less-affected side. We indeed observed these predicted changes with bilateral stimulation.

To compare potential effects of unilateral versus bilateral stimulation, we used the computational model of Truong et al. (2014) and estimated the electric field that was produced during $2 \mathrm{~mA}$ of bilateral stimulation over M1 (Fig. 10, top row). The electric field generated by bilateral stimulation (one electrode placed over C3 and the other over C4) engaged both the frontal and parietal lobes in each hemisphere. It had its peak intensity over the motor cortex (peak intensity at $-16,-25,+55$, Talairach coordinates, peak electric field of $0.716 \mathrm{~V} / \mathrm{m}$, peak current of $0.215 \pm 0.05 \mathrm{~A} / \mathrm{m}^{2}$ ). In comparison, when one electrode was placed over M1 (location C3) and the other over the supraorbital 


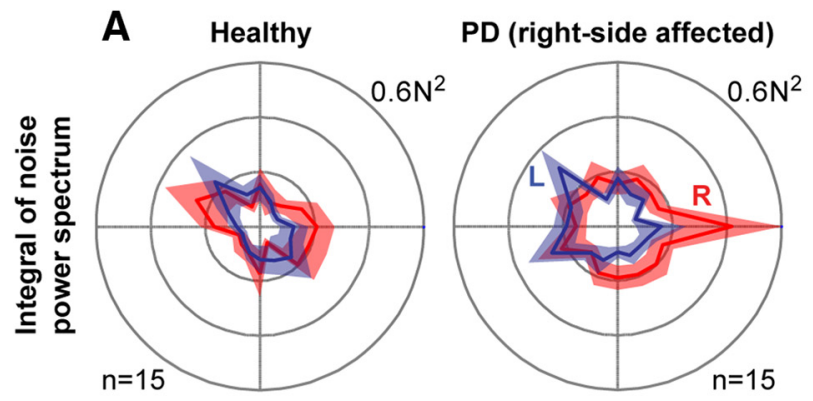

B

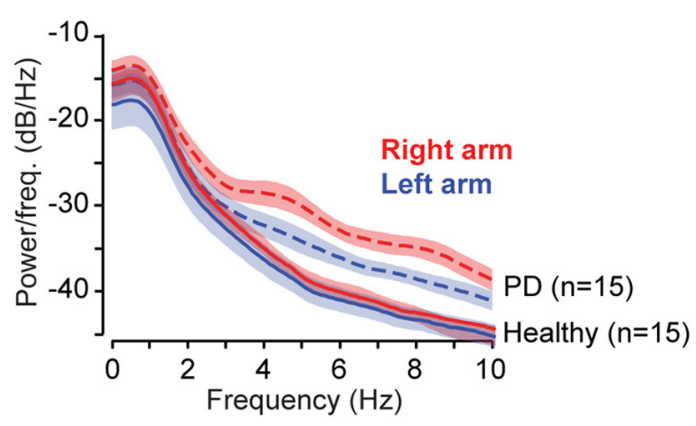

C

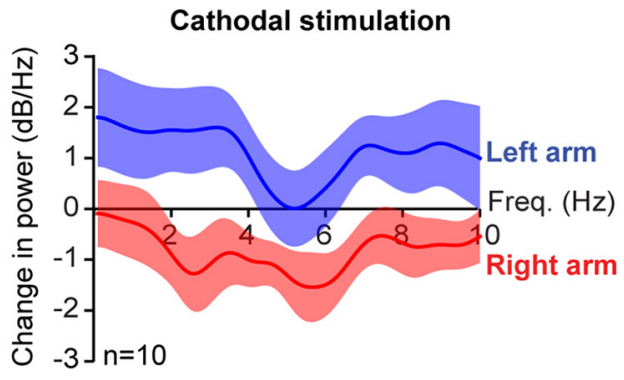

Figure 9. Power spectrum of noise as measured in the unimanual task and the effects of cortical stimulation. For each subject, we computed the power spectrum for each instructed force direction and amplitude. $\boldsymbol{A}$, Data are for subjects in Experiment 1. The power spectrum for each subject was averaged across force magnitudes in each direction. Noise is directiondependent in PD patients and larger for the right side in quadrants 1 and 4. B, Data are for subjects in Experiment 1. The power spectrum for each subject was averaged across force magnitudes and directions. Noise is larger for the PD patients, particularly in the higher frequencies. C, Data for subjects in Experiment 3, cathodal stimulation of the left M1 (anode placed on the right $\mathrm{M1}$ ). Error bars indicate SEM.

region of the contralateral side (location FP2), the peak of the electric field was over the premotor cortex and more anterior regions (peak intensity at $-15,-10,+45$, Talairach coordinates, with peak electric field of $0.706 \mathrm{~V} / \mathrm{m}$, peak current of $0.212 \pm 0.05$ $\mathrm{A} / \mathrm{m}^{2}$ ). This suggests that bilateral stimulation may be a better approach for stimulating the affected M1.

\section{Discussion}

We used a bimanual task in which people with PD chose how much force to produce with each arm in order for the sum to equal a target amount and made the following observations. First, despite the fact that the strengths of the two arms were normal, the PD patients preferred to assign most of the force to the lessaffected arm. However, this preference was direction dependent: for some directions, the patient shared the burden between the two arms, whereas for other directions, the patient assigned the burden almost exclusively to the less-affected arm. The combination of choices and strengths of each arm illustrated that the subjective cost of force production was abnormally large for only certain directions. Second, in a unimanual task, we measured the signal-dependent noise in each arm and found that, unlike healthy controls, noise in PD was lateralized, generally higher on the affected side. The directions for which the patients were less willing to assign force to an arm were generally the directions for which that arm had greater unimanual noise. Finally, we improved the willingness of patients to assign force to the affected arm via noninvasive bilateral stimulation. Placing the cathode over the motor cortex contralateral to the affected arm and the anode over the less-affected side reduced the lateralization of noise, increased the willingness to assign force to the affected arm, and produced significant reductions in clinical motor symptoms of the disease.

\section{Subjective cost of force production in PD patients}

Dopamine depletion does not alter the liking of the rewarding stimulus (e.g., food), but rather reduces the willingness of animals to work for that food (Salamone and Correa, 2002). Because of the asymmetric loss of dopamine in PD, this view would suggest that given the option, the PD brain would rather produce the force with the less-affected arm. Our study confirmed this prediction, but uncovered the surprising result that the preference was direction dependent; for some directions, the force assigned to each arm was normal, whereas for other directions, particularly along directions that engaged shoulder and elbow extensor muscles on the more-affected side (Fig. 2C), the forces were grossly lateralized to the less-affected arm. This suggested that loss of dopamine coincided with direction-dependent increases in the subjective cost of force production on the contralateral arm.

In an earlier work, PD patients were asked to use their hand on the more-affected side to produce a reference force and then match that force with the other hand (Lafargue et al., 2008). The patients produced only half of the reference force with the lessaffected side; that is, on average, the subjective effort that they perceived on the more-affected side was approximately twice that of the effort they perceived on the less-affected side. In our task, we found that some directions of force carried an effort cost that was up to five times higher on the affected side, whereas other directions of force generation carried a normal effort cost. This direction-dependent pattern was largely explained by lateralization of unimanual noise: the cost was higher in a given direction if the noise in that direction was larger.

Signal-dependent noise in PD patients has been measured in an isometric force production task that engaged finger muscles (Dengler et al., 1986). The motor units of the PD patients showed increased SD of action potential timing during force production. Earlier work had also noted that single joint movements in PD appeared to be more affected during extension than during flexion (Agostino et al., 2003; Robichaud et al., 2004). Furthermore, in $\mathrm{PD}$, rigidity is generally higher for extensor muscles of the wrist than flexor muscles (Xia et al., 2006). Together, control of extensor muscles may be more strongly compromised than flexor muscles, which may contribute to the direction-dependent increase in the subjective cost of force.

A fundamental prediction of optimal control models of motor control (Todorov and Jordan, 2002) is that the brain should avoid assigning actions to actors that are noisy (Shadmehr and Mussa-Ivaldi, 2012). Our results here suggest that, in both healthy subjects and PD patients, the brain attempts to produce motor commands that minimize a simple cost, one that penalizes motor commands based on strength and noise. In PD patients, part of the increased cost of force production can be accounted for by increased noise. 

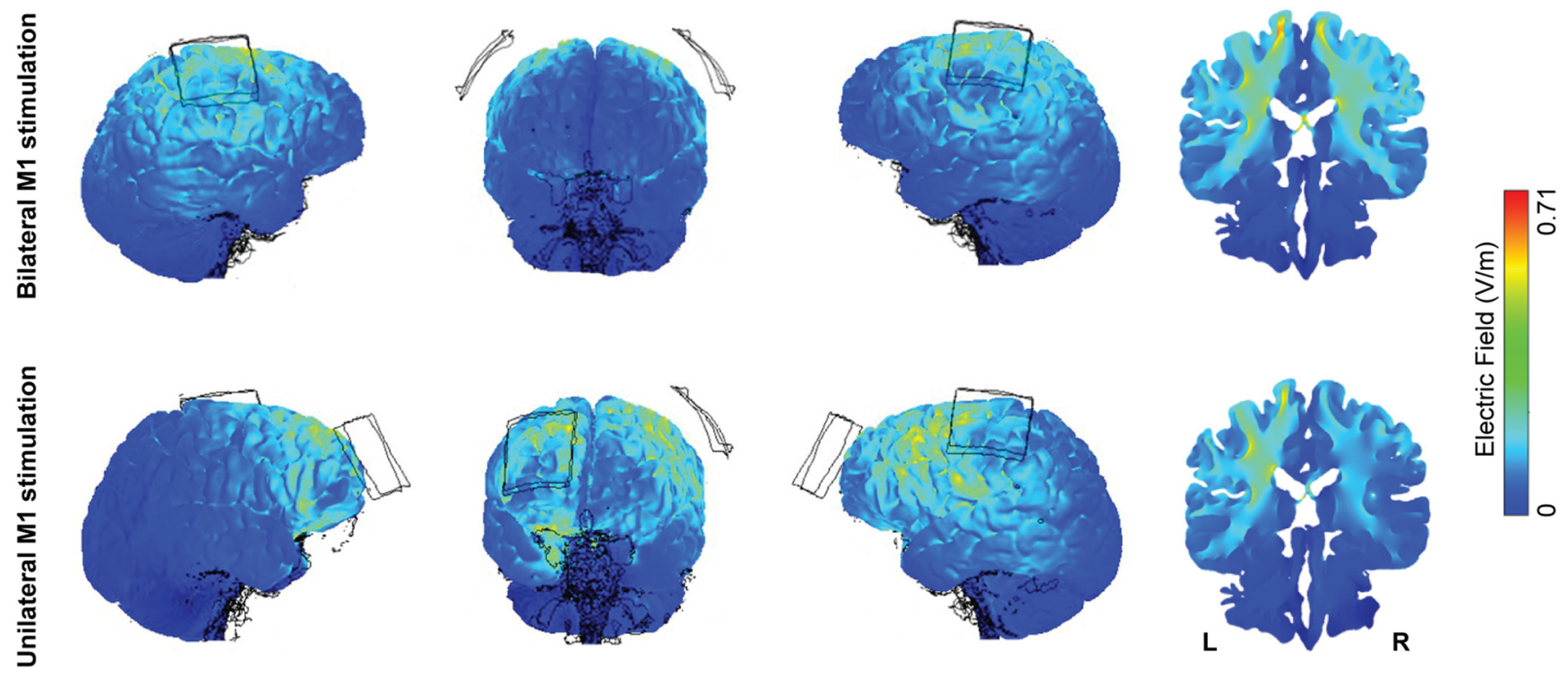

Figure 10. The electric field generated by placement of the tDCS electrodes bilaterally on $\mathrm{M} 1$ (top row) or unilaterally (bottom row). The electrodes are $5 \times 5 \mathrm{~cm}$ in size and are shown by the square regions centered over the 33 and $C 4$ locations of the EEG 10-20 system. In the unilateral montage, one electrode is placed on the left M1 (C3 location) and the other electrode is placed on the right supraorbital region. The cornel view (right column) is a slice through C3-C4 region. Simulation results are for $2 \mathrm{~mA}$ current based on the methods described in Truong et al. (2014).

\section{Effects of tDCS on dopamine}

How might tDCS alter the subjective cost of force production? In a recent study in anesthetized rats, Tanaka et al. (2013) used microdialysis to quantify extracellular dopamine in the striatum. They reported that, after $10 \mathrm{~min}$ of cathodal tDCS of the frontal lobe, there was a $60 \%$ increase in dopamine concentration in the ipsilateral striatum, an increase that was sustained for hours after cessation of stimulation. Importantly, this increase was specific to cathodal stimulation because anodal stimulation produced a $10 \%$ decrease in dopamine. Therefore, it is possible that, in our study, cathodal stimulation over M1 indirectly enhanced dopamine release in the ipsilateral striatum.

In theory, an increased relative basal level of striatal dopamine contralateral to the affected side can account for general reductions in subjective cost of force production. Hwang (2013) has proposed that striatal cells that are part of the indirect pathway of the basal ganglia may be involved in the encoding of effort associated with a planned action. In this framework, increased striatal dopamine would reduce the activity in the indirect pathway, increasing the willingness to choose actions associated with the contralateral side (Tai et al., 2012).

Our patients were on their normal schedule of medication and this is likely to have played an important role in the effects of stimulation. A key finding is regarding the interaction of tDCS with basal levels of dopamine. In healthy individuals, anodal tDCS over M1 makes the neurons easier to excite because it brings them closer to depolarization threshold (Fritsch et al., 2010). However, when healthy people are given L-DOPA to increase basal levels of dopamine, the effects of anodal tDCS are blunted, eliminating the potentiation (Kuo et al., 2008). That is, in healthy people, increased basal levels of dopamine make anodal tDCS ineffective in potentiating the cortical neurons. This interaction of anodal stimulation with basal levels of dopamine may explain the fact that we and others have not observed positive effects of anodal tDCS in medicated PD patients (Benninger et al., 2010; Verheyden et al., 2013; but see Valentino et al., 2014). In contrast, in off-medication patients, Fregni et al. (2006) reported that both anodal and cathodal stimulation produced improvements in a pegboard task, but the effects of anodal stimulation were larger.

\section{Stimulation over M1}

We placed the electrodes over M1 because, during isometric force production, cells in M1 are strongly modulated by the direction and magnitude of force (Sergio and Kalaska, 1998; Cabel et al., 2001), whereas modulation is smaller in other frontal motor areas (Kakei et al., 2001) and much less in the posterior parietal cortex (Kalaska et al., 1990). That is, force production tends to strongly engage the cells in M1.

In a previous study, we found that the unimanual noise patterns had a directional dependence that agreed with the distribution of preferred direction of cells in M1, as shown in Figure 8 of Salimpour and Shadmehr (2014): in healthy people, directions of force production that engaged a greater number of cells in the motor cortex coincided with directions of lower noise in the resulting force. This also suggested that the task that we considered depended on properties of M1.

\section{Limitations}

We did not measure the relationship between subjective cost of force production and reward. A study approached this question by providing fictitious monetary rewards in exchange for force production (Schmidt et al., 2008) and found that the slope of the function relating force to money was shallower than normal in PD patients. That is, a given increase in reward produced a smaller than normal increase in the willingness to produce force in $\mathrm{PD}$ patients.

Our study was not a clinical trial, so the fact that we found significant reductions in clinical motor symptoms must be taken with caution. However, our results identify a new avenue of research for treatment of PD. Unimanual noise can be improved using behavioral training techniques (Shmuelof et al., 2012) and the behavioral outcome of these techniques may be enhanced with tDCS (Reis et al., 2009).

We focused on one montage of stimulation (bilateral M1) and measured the effects of altering stimulation at that location, but 
did not try other locations of stimulation. Therefore, we do not know if this is the optimal placement of the electrodes. A more accurate placement of the electrodes, perhaps through an MRIguided technique or the use of TMS, or placement of the electrodes at other locations may produce better results.

Our approach took advantage of the fact that PD motor symptoms are lateralized (Gelb et al., 1999). Lateralization is a stable feature of PD (Toth et al., 2004) that is maintained with disease progression (Lee et al., 1995), but varies among patients.

There is now a body of data suggesting the potential efficacy of tDCS in reducing cognitive and motor symptoms of PD (Doruk et al., 2014; Valentino et al., 2014). Here, we demonstrated that, by altering noise via tDCS, it was possible to change the brain's willingness to exert effort with the affected limb. The fact that these effects were observed in medicated patients, whose dopamine stimulation was optimized, may be of clinical relevance.

\section{References}

Agostino R, Currà A, Giovannelli M, Modugno N, Manfredi M, Berardelli A (2003) Impairment of individual finger movements in Parkinson's disease. Mov Disord 18:560-565. CrossRef Medline

Ballanger B, Thobois S, Baraduc P, Turner RS, Broussolle E, Desmurget M (2006) "Paradoxical kinesis" is not a hallmark of Parkinson's disease but a general property of the motor system. Mov Disord 21:1490-1495. CrossRef Medline

Bardgett ME, Depenbrock M, Downs N, Points M, Green L (2009) Dopamine modulates effort-based decision making in rats. Behav Neurosci 123:242-251. CrossRef Medline

Benninger DH, Lomarev M, Lopez G, Wassermann EM, Li X, Considine E, Hallett M (2010) Transcranial direct current stimulation for the treatment of Parkinson's disease. J Neurol Neurosurg Psychiatry 81:11051111. CrossRef Medline

Cabel DW, Cisek P, Scott SH (2001) Neural activity in primary motor cortex related to mechanical loads applied to the shoulder and elbow during a postural task. J Neurophysiol 86:2102-2108. Medline

Correa M, Carlson BB, Wisniecki A, Salamone JD (2002) Nucleus accumbens dopamine and work requirements on interval schedules. Behav Brain Res 137:179-187. CrossRef Medline

Craigmile PF, Guttorp P, Percival DB (2005) Wavelet-based parameter estimation for polynomial contaminated fractionally differenced processes. IEEE Trans Sig Proc 53:3151-3161. CrossRef

Dengler R, Wolf W, ScHübert M, Struppler A (1986) Discharge pattern of single motor units in basal ganglia disorders. Neurology 36:1061-1066. CrossRef Medline

Doruk D, Gray Z, Bravo GL, Pascual-Leone A, Fregni F (2014) Effects of tDCS on executive function in Parkinson's disease. Neurosci Lett 582: 27-31. CrossRef Medline

Floresco SB, Tse MT, Ghods-Sharifi S (2008) Dopaminergic and glutamatergic regulation of effort- and delay-based decision making. Neuropsychopharmacology 33:1966-1979. CrossRef Medline

Fregni F, Boggio PS, Santos MC, Lima M, Vieira AL, Rigonatti SP, Silva MT, Barbosa ER, Nitsche MA, Pascual-Leone A (2006) Noninvasive cortical stimulation with transcranial direct current stimulation in Parkinson's disease. Mov Disord 21:1693-1702. CrossRef Medline

Fritsch B, Reis J, Martinowich K, Schambra HM, Ji Y, Cohen LG, Lu B (2010) Direct current stimulation promotes BDNF-dependent synaptic plasticity: potential implications for motor learning. Neuron 66:198-204. CrossRef Medline

Gelb DJ, Oliver E, Gilman S (1999) Diagnostic criteria for Parkinson disease. Arch Neurol 56:33-39. CrossRef Medline

Goertzel G (1958) An algorithm for the evaluation of finite trigonometric series. The American Mathematical Monthly 65:34-35. CrossRef

Hwang EJ (2013) The basal ganglia, the ideal machinery for the cost-benefit analysis of action plans. Front Neural Circuits 7:121. Medline

Kakei S, Hoffman DS, Strick PL (2001) Direction of action is represented in the ventral premotor cortex. Nat Neurosci 4:1020-1025. CrossRef Medline

Kalaska JF, Cohen DA, Prud'homme M, Hyde ML (1990) Parietal area 5 neuronal activity encodes movement kinematics, not movement dynamics. Exp Brain Res 80:351-364. Medline
Kuo MF, Paulus W, Nitsche MA (2008) Boosting focally-induced brain plasticity by dopamine. Cereb Cortex 18:648-651. CrossRef Medline

Lafargue G, D'Amico A, Thobois S, Broussolle E, Sirigu A (2008) The ability to assess muscular force in asymmetrical Parkinson's disease. Cortex 44: 82-89. CrossRef Medline

Lee CS, Schulzer M, Mak E, Hammerstad JP, Calne S, Calne DB (1995) Patterns of asymmetry do not change over the course of idiopathic parkinsonism: implications for pathogenesis. Neurology 45:435-439. CrossRef Medline

Majsak MJ, Kaminski T, Gentile AM, Flanagan JR (1998) The reaching movements of patients with Parkinson's disease under self-determined maximal speed and visually cued conditions. Brain 121:755-766. CrossRef Medline

Mazzoni P, Hristova A, Krakauer JW (2007) Why don't we move faster? Parkinson's disease, movement vigor, and implicit motivation. J Neurosci 27:7105-7116. CrossRef Medline

Oldfield RC (1971) The assessment and analysis of handedness: the Edinburgh inventory. Neuropsycholgia 9:97-113. CrossRef Medline

O'Sullivan I, Burdet E, Diedrichsen J (2009) Dissociating variability and effort as determinants of coordination. PLoS Comput Biol 5:e1000345. CrossRef Medline

Peto V, Jenkinson C, Fitzpatrick R, Greenhall R (1995) The development and validation of a short measure of functioning and well being for individuals with Parkinson's disease. Qual Life Res 4:241-248. CrossRef Medline

Reis J, Schambra HM, Cohen LG, Buch ER, Fritsch B, Zarahn E, Celnik PA, Krakauer JW (2009) Noninvasive cortical stimulation enhances motor skill acquisition over multiple days through an effect on consolidation. Proc Natl Acad Sci U S A 106:1590-1595. CrossRef Medline

Robichaud JA, Pfann KD, Comella CL, Brandabur M, Corcos DM (2004) Greater impairment of extension movements as compared to flexion movements in Parkinson's disease. Exp Brain Res 156:240-254. CrossRef Medline

Salamone JD, Correa M (2002) Motivational views of reinforcement: implications for understanding the behavioral functions of nucleus accumbens dopamine. Behav Brain Res 137:3-25. CrossRef Medline

Salamone JD, Steinpreis RE, McCullough LD, Smith P, Grebel D, Mahan K (1991) Haloperidol and nucleus accumbens dopamine depletion suppress lever pressing for food but increase free food consumption in a novel food choice procedure. Psychopharmacology (Berl) 104:515-521. CrossRef Medline

Salimpour Y, Shadmehr R (2014) Motor costs and the coordination of the two arms. J Neurosci 34:1806-1818. CrossRef Medline

Schmidt L, d'Arc BF, Lafargue G, Galanaud D, Czernecki V, Grabli D, Schüpbach M, Hartmann A, Levy R, Dubois B, Pessiglione M (2008) Disconnecting force from money: effects of basal ganglia damage on incentive motivation. Brain 131:1303-1310. Medline

Scott SH, Gribble PL, Graham KM, Cabel DW (2001) Dissociation between hand motion and population vectors from neural activity in motor cortex. Nature 413:161-165. CrossRef Medline

Sergio LE, Kalaska JF (1998) Changes in the temporal pattern of primary motor cortex activity in a directional isometric force versus limb movement task. J Neurophysiol 80:1577-1583. Medline

Shadmehr R, Mussa-Ivaldi S (2012) Biological learning and control: how the brain builds representations, predicts events, and makes decisions. Cambridge, MA: MIT.

Shmuelof L, Krakauer JW, Mazzoni P (2012) How is a motor skill learned? Change and invariance at the levels of task success and trajectory control. J Neurophysiol 108:578-594. CrossRef Medline

Tai LH, Lee AM, Benavidez N, Bonci A, Wilbrecht L (2012) Transient stimulation of distinct subpopulations of striatal neurons mimics changes in action value. Nat Neurosci 15:1281-1289. CrossRef Medline

Tanaka T, Takano Y, Tanaka S, Hironaka N, Kobayashi K, Hanakawa T, Watanabe K, Honda M (2013) Transcranial direct-current stimulation increases extracellular dopamine levels in the rat striatum. Front Syst Neurosci 7:6. Medline

Tatsch K, Schwarz J, Mozley PD, Linke R, Pogarell O, Oertel WH, Fieber RS, Hahn K, Kung HF (1997) Relationship between clinical features of Parkinson's disease and presynaptic dopamine transporter binding assessed with [123I]IPT and single-photon emission tomography. Eur J Nucl Med 24:415-421. Medline 
Todorov E, Jordan MI (2002) Optimal feedback control as a theory of motor coordination. Nat Neurosci 5:1226-1235. CrossRef Medline

Toth C, Rajput M, Rajput AH (2004) Anomalies of asymmetry of clinical signs in parkinsonism. Mov Disord 19:151-157. CrossRef Medline

Truong DQ, Hüber M, Xie X, Datta A, Rahman A, Parra LC, Dmochowski JP, Bikson M (2014) Clinician accessible tools for GUI computational models of transcranial electrical stimulation: BONSAI and SPHERES. Brain Stimul 7:521-524. CrossRef Medline

Valentino F, Cosentino G, Brighina F, Pozzi NG, Sandrini G, Fierro B, Savettieri G, D'Amelio M, Pacchetti C (2014) Transcranial direct current stimulation for treatment of freezing of gait: a cross-over study. Mov Disord 29:1064-1069. CrossRef Medline van der Hoorn A, Burger H, Leenders KL, de Jong BM (2012) Handedness correlates with the dominant Parkinson side: a systematic review and meta-analysis. Mov Disord 27:206-210. CrossRef Medline

Verheyden G, Purdey J, Burnett M, Cole J, Ashburn A (2013) Immediate effect of transcranial direct current stimulation on postural stability and functional mobility in Parkinson's disease. Mov Disord 28:2040-2041. CrossRef Medline

Wardle MC, Treadway MT, Mayo LM, Zald DH, de Wit H (2011) Amping up effort: effects of d-amphetamine on human effort-based decisionmaking. J Neurosci 31:16597-16602. CrossRef Medline

Xia R, Markopoulou K, Puumala SE, Rymer WZ (2006) A comparison of the effects of imposed extension and flexion movements on Parkinsonian rigidity. Clin Neurophysiol 117:2302-2307. CrossRef Medline 